\title{
ON CUBIC HYPERSURFACES WITH VANISHING HESSIAN
}

\author{
RODRIGO GONDIM AND FRANCESCO RUSSO*
}

\begin{abstract}
We prove that for $N \leq 6$ an irreducible cubic hypersurface with vanishing hessian in $\mathbb{P}^{N}$ is either a cone or a scroll in linear spaces tangent to the dual of the image of the polar map of the hypersurface. We also provide canonical forms and a projective characterization of Special Perazzo Cubic Hypersurfaces, which, a posteriori, exhaust the class of cubic hypersurfaces with vanishing hessian, not cones, for $N \leq 6$. Finally we show by pertinent examples the technical difficulties arising for $N \geq 7$.
\end{abstract}

\section{INTRODUCTION}

The aim of this paper is to provide the classification of cubic hypersurfaces with vanishing hessian in $\mathbb{P}^{N}$ for $N \leq 6$ (see Theorems 4.2, 4.3, 4.4, 4.6, 4.7).

Hypersurfaces with vanishing hessian were studied systematically for the first time in the fundamental paper [GN], where Gordan and M. Noether analyze Hesse's claims in [He1, He2] according to which these hypersurfaces are necessarily cones.

More explicitly, if $X=V(f) \subset \mathbb{P}^{N}$ is a reduced complex hypersurface, the hessian of $f$ (or by abusing the terminology the hessian of $X$ ), indicated by hess ${ }_{X}$, is the determinant of the matrix of the second derivatives of the form $f$, that is the determinant of the so called hessian matrix of $f$.

Of course cones have vanishing hessian and Hesse claimed twice in [He1] and in [He2] that a hypersurface $X \subset \mathbb{P}^{N}$ is a cone if $\operatorname{hess}_{X}=0$. Clearly the claim is true if $\operatorname{deg}(X)=2$ so that the first relevant case for the problem is that of cubic hypersurfaces. One immediately sees that $V\left(x_{0} x_{3}^{2}+x_{1} x_{3} x_{4}+x_{2} x_{4}^{2}\right) \subset \mathbb{P}^{4}$ is a cubic hypersurface with vanishing hessian but not a cone, for example because the first partial derivatives of the equation are linearly independent.

Actually the question is quite subtle because, as it was firstly pointed out in [GN], the claim is true for $N \leq 3$ and in general false for $N \geq 4$. The cases $N=1,2$ are easily handled but beginning from $N=3$ the question is related to non trivial characterizations of cones among developable hypersurfaces or, from a differential point of view, to a characterization of algebraic cones (or of algebraic cylinders in the affine setting) among hypersurfaces with zero gaussian curvature at every regular point.

Gordan and Noether approach to the problem and their proofs for $N \leq 3$ have been revisited recently in modern terms in [Lo] and [GR], see also [Pt1, Pt2]. In [GN] it is constructed a series of projective hypersurfaces in $\mathbb{P}^{N}$ for every $N \geq 4$, which generically are not cones and to which the explicit example recalled above belongs, see also [Pt1, Pt2, CRS, Section 2] and Example 1. Moreover Gordan and Noether also classified all hypersurfaces

*Partially supported by the PRIN "Geometria delle varietà algebriche"; the author is a member of the G.N.S.A.G.A. 
with vanishing hessian for $N \leq 4$ proving that they are either cones or $N=4$ and the hypersurfaces belong to their series of examples, see loc. cit., Fr2 and GR. The work of Perazzo considered the classification of cubic hypersurfaces with vanishing hessian for $N \leq 6$, see [Pe, and analyzed particular subclasses dubbed by us Special Perazzo Cubic Hypersurfaces. Perazzo's ideas and techniques are very interesting and inspired deeply our work although in our opinion they contain some serious mistakes and non motivated claims which unfortunately affect most of the main results, see Remark 2.6 for a discussion of some of these imprecise statements.

As far as we know no explicit classification result is known for $N \geq 5$ so that our contribution is the first attempt to extend to higher dimension Gordan-Noether-Franchetta classification of hypersurfaces with vanishing hessian albeit only in the cubic case.

Hypersurfaces with vanishing hessian remained outside the mainstream of (algebraic) geometry for a long time although they represent very interesting objects for many areas of research. For example the cubic hypersurface recalled above, very well known to classical algebraic geometers, is celebrated in the modern algebraic-differential geometry literature as the Bourgain-Sacksteder Hypersurface (see [AG2, AG1, FP]). Moreover the regular points of a hypersurface with vanishing hessian are all parabolic and represent a natural generalization of the flex points of plane curves (see [Ci, CRS]). The divisibility properties of the hessian with respect to the original form have interesting geometric consequences, see [Se1, Ci]; for instance developable hypersurfaces are those for which hess $x_{X}=0(\bmod f)$, see [Se1, AG1, FP].

We now describe the sections of the paper in more detail. In Section 1 we introduce the notation, recall some well known results and define the polar map of a hypersurface. We also state without proof the Gordan-Noether Identity for hypersurfaces with vanishing hessian and deduce some important geometrical consequences in Theorem 1.9 and Corollary 1.10. In Section 2 we introduce the Perazzo map of a hypersurface with vanishing hessian following $[\mathrm{Pe}$ and then we study the geometry associated to the fibers of this map and their relation with the key variety $Z^{*}$, which is the dual of the closure of the image of the polar map, see Section 1 for precise definitions. Firstly we prove that the closure of a general fiber of the Perazzo map is a linear space, Theorem 2.5, correcting some mistakes contained in $[\mathrm{Pe}$, see Remark 2.6 and Proposition 2.7. Then we analyze the congruence of linear spaces determined by the fibers of the Perazzo map introducing the notion of Special Perazzo Cubic Hypersurface which corresponds to the case in which the congruence of the fibers of the Perazzo map is the family of linear spaces passing through a fixed (codimension one) linear space, see Proposition 2.12 .

Special Perazzo Cubic Hypersurfaces are somehow surprising since they are ruled by a family of linear spaces along which the hypersurface is not developable so that this ruling is different from the one given by the fibers of the Gauss map. These examples and their generalizations are known in differential geometry as twisted planes, see for example [FP]. Despite the huge number of papers dedicated to this subject in differential geometry very few classification or structure results have been obtained. In our opinion the global point of view provided by polarity, which has been overlooked until now by differential geometers, is a stronger tool to treat these objects.

In Section 3 we exhibit canonical forms of cubics with vanishing hessian and singular along a linear space and we also prove a projective characterization of Special Perazzo Cubic Hypersurfaces following [Pe. Section 4 contains the classification and structure of cubic 
hypersurfaces with vanishing hessian having $\operatorname{dim}\left(Z^{*}\right)=1$, Theorem 4.1. With these results the classification of cubic hypersufaces with vanishing hessian in $\mathbb{P}^{N}, N \leq 6$, is easily deduced in the rest of Section 4. In Section 5 we discuss some examples showing the difficulties arising for $N \geq 7$ and the existence of some exceptional and sporadic phenomena.

\section{Preliminaries}

1.1. Cones, Hessian and the Polar Map of a Hypersurface. Let $X=V(f) \subset \mathbb{P}^{N}$ be a reduced hypersurface and let $d=\operatorname{deg}(X) \geq 1$ be its degree. From now on we shall also restrict to the case of an algebraically closed field $\mathbb{K}$ of characteristic zero.

For a set $S \subseteq \mathbb{P}^{N}$ we shall indicate by $\left\langle S>\right.$ its linear span in $\mathbb{P}^{N}$ and $S \subseteq \mathbb{P}^{N}$ is said to be degenerate if $\left\langle S>\subsetneq \mathbb{P}^{N}\right.$. With this notation $\langle p, q\rangle$ is the line through two distinct points $p, q \in \mathbb{P}^{N}$.

Definition 1.1. Let $X \subset \mathbb{P}^{N}$ be a projective variety. The vertex of $X$ is

$$
\operatorname{Vert}(X)=\{p \in X \mid<p, q>\subset X, \forall q \in X\} .
$$

A projective variety $X \subset \mathbb{P}^{N}$ is a cone if $\operatorname{Vert}(X) \neq \emptyset$.

Remark 1.2. The set $\operatorname{Vert}(X) \subset \mathbb{P}^{N}$ is a linear subspace and, by generic smoothness, $\operatorname{Vert}(X)=\bigcap_{x \in X} T_{x} X$, where $T_{x} X$ is the projective tangent space to $X$ at $x$.

Let $X \subset \mathbb{P}^{N}$ be an equidimensional variety of dimension $n=\operatorname{dim}(X) \geq 1$. Then $\operatorname{dim}(\operatorname{Vert}(X)) \geq n-1$ implies that $X$ is the union of linear spaces passing through $\operatorname{Vert}(X)$.

We present, without proof, some well known results used in the sequel.

Proposition 1.3. Let $X=V(f) \subset \mathbb{P}^{N}$ be a hypersurface of degree $d$. Then the following conditions are equivalent:

i) $X$ is a cone;

ii) There exists a point $p \in X$ of multiplicity $d$;

iii) The partial derivatives $\frac{\partial f}{\partial x_{0}}, \frac{\partial f}{\partial x_{1}}, \ldots, \frac{\partial f}{\partial x_{N}}$ of $f$ are linearly dependent;

iv) Up to a projective transformation, $f$ depends on at most $N$ variables.

v) The dual variety of $X, X^{*} \subset\left(\mathbb{P}^{N}\right)^{*}$, is degenerate.

Definition 1.4. Let $X=V(f) \subset \mathbb{P}^{N}$ be a reduced hypersurface. The Hessian matrix of $f$ is

$$
\operatorname{Hess}_{f}=\left[\frac{\partial^{2} f}{\partial x_{i} \partial x_{j}}\right]_{0 \leq i, j \leq N}
$$

We also call it the hessian matrix of $X$ and write $\operatorname{Hess}_{X}$ since we will be interested in properties of this matrix (like the vanishing of its determinant or more generally its rank) which are well defined modulo the multiplication of $f$ by a non-zero constant. The determinant of the matrix $\operatorname{Hess}_{X}$ will be denoted by hess $X$ and called the hessian of $X$. Thus it is defined modulo a non zero constant and it is a projective covariant whose vanishing does not depend on the equation of $X$.

Cones form a trivial class of hypersurfaces with vanishing hessian. Indeed if $X$ is a cone up to a linear change of coordinates the form $f$ does not depend on all the variables, see also Proposition 1.3. Therefore in this case the Hessian matrix has at least a null row (and a null column), yielding hess $X=0$. 
The converse is not true in general if $d \geq 3$ as shown by the example recalled in the Introduction. We now define the polar map of a hypersurface in order to begin to clarify the deep geometrical consequences of the condition hess ${ }_{X}=0$.

Definition 1.5. The polar map (or gradient map) of a hypersurface $X=V(f) \subset \mathbb{P}^{N}$, indicated by $\Phi_{X}$ or by $\Phi_{f}$, is the rational map $\Phi_{X}: \mathbb{P}^{N} \rightarrow\left(\mathbb{P}^{N}\right)^{*}$ given by the derivatives of $f$ :

$$
\Phi_{X}(p)=\left(\frac{\partial f}{\partial x_{0}}(p): \frac{\partial f}{\partial x_{1}}(p): \ldots: \frac{\partial f}{\partial x_{N}}(p)\right) .
$$

Let $Z=\overline{\Phi_{X}\left(\mathbb{P}^{N}\right)} \subseteq\left(\mathbb{P}^{N}\right)^{*}$ be the closure of the image of the polar map. The base locus scheme of the polar map is the singular scheme of $X$ which will be denoted by

$$
\text { Sing } X:=V\left(\frac{\partial f}{\partial x_{0}}, \ldots, \frac{\partial f}{\partial x_{N}}\right) \subset \mathbb{P}^{N} .
$$

We shall maintain it distinct from the set theoretic singular locus ( $\operatorname{Sing} X)_{\text {red }}$.

If $p=[\mathbf{v}]$, then $t_{p} \mathbb{P}^{N}=\mathbb{K}^{N+1} /<\mathbf{v}>$ is the affine tangent space to $\mathbb{P}^{N}$ at $p$. Let $\operatorname{Hess}_{X}(p)$ be the equivalence class of the hessian matrix of $X$ evaluated at $\mathbf{v}$. By Euler's formula the equivalence class of the linear $\operatorname{map} \operatorname{Hess}_{X}(p)$ passes to the quotients and it induces the differential of the map $\Phi_{X}$ at $p$ :

$$
\left(d \Phi_{X}\right)_{p}: t_{p} \mathbb{P}^{N} \rightarrow t_{\Phi_{X}(p)} \mathbb{P}^{N}
$$

whose image is $t_{\Phi_{X}(p)} Z$ if $p$ is general by generic smoothness. From this we can describe the projective tangent space to $Z$ at $\Phi_{X}(p)$ for $p \in \mathbb{P}^{N}$ general, obtaining

$$
T_{\Phi_{X}(p)} Z=\mathbb{P}\left(\operatorname{Im}\left(\operatorname{Hess}_{X}(p)\right)\right) \subseteq\left(\mathbb{P}^{N}\right)^{*} .
$$

Thus

$$
\operatorname{dim} Z=\operatorname{rk}\left(\operatorname{Hess}_{X}\right)-1
$$

Therefore hess $\operatorname{hos}_{X}=0$ if and only if $Z \subsetneq \mathbb{P}^{N *}$ if and only if the partial derivatives of $f$ are algebraically dependent, that is there exists a non zero homogeneous polynomial $g \in$ $\mathbb{K}\left[y_{0}, \ldots, y_{N}\right]$ such that $g\left(\frac{\partial f}{\partial x_{0}}, \ldots, \frac{\partial f}{\partial x_{N}}\right)=0$.

From this perspective Hesse's claim can be translated into asking if the algebraic dependence of the first partial derivatives of a homogeneous form implies their linear dependence, a quite subtle question as we mentioned in the Introduction and whose answer is negative in general but positive for $N \leq 3$, see loc. cit.

The next result will be important to describe the structure of hypersurfaces with vanishing hessian via the analysis of the restriction of the polar map to a hyperplane, for a proof see [CRS, Lemma 3.10].

Lemma 1.6. Let $X=V(f) \subset \mathbb{P}^{N}$ be a hypersurface. Let $H=\mathbb{P}^{N-1}$ be a hyperplane not contained in $X$, let $h=H^{*}$ be the corresponding point in $\mathbb{P}^{n *}$ and let $\pi_{h}$ denote the projection from the point $h$. Then:

$$
\Phi_{V(f) \cap H}=\pi_{h} \circ\left(\Phi_{f \mid H}\right) .
$$

In particular, $Z(V(f) \cap H) \subseteq \pi_{h}(Z(f))$, where $Z(V(f) \cap H)$ denotes the closure of the image of the polar map $\Phi_{V(f) \cap H}: H=\mathbb{P}^{n-1} \rightarrow\left(\mathbb{P}^{n-1}\right)^{*}$. 
Definition 1.7. Let $X \subset \mathbb{P}^{N}$ be a reduced equidimensional projective variety of dimension $\operatorname{dim} X=n$. The Gauss map of $X, \mathcal{G}_{X}: X \rightarrow \mathbb{G}(n, N)$, is the rational map associating to every smooth point of $X$ its embedded projective tangent space considered as a point in $\mathbb{G}(n, N)$, i.e. $\mathcal{G}_{X}(p)=\left[T_{p} X\right] \in \mathbb{G}(n, N)$.

We shall later consider also the dual Gauss map of $X$ defined at a smooth point $x \in X$ by $\mathcal{G}_{X}^{*}(x)=\left[\left(T_{x} X\right)^{*}\right] \in \mathbb{G}(N-n-1, N)$.

In the special case of hypersurfaces, $X=V(f) \subset \mathbb{P}^{N}$, the Gauss map of $X$ is the restriction of the polar map of $X$ to $X$ and the image of the Gauss map is the dual variety $X^{*}$. In particular $X^{*} \subseteq Z$.

1.2. The Gordan-Noether Identity and its geometrical consequences. From now on $f \in \mathbb{K}\left[x_{0}, \ldots, x_{N}\right]$ will be a homogeneous reduced polynomial of degree $d$ such that hess $f=0$, unless otherwise stated.

Since hess $f_{f}=0$, there exist non zero homogeneous polynomials $\pi \in \mathbb{K}\left[y_{0}, \ldots, y_{N}\right]$ such that $\pi\left(\frac{\partial f}{\partial x_{0}}, \ldots, \frac{\partial f}{\partial x_{N}}\right)=0$. Let $g \in \mathbb{K}\left[y_{0}, \ldots, y_{N}\right]$ be an irreducible polynomial with this property and such that $g_{i}:=\frac{\partial g}{\partial y_{i}}\left(\frac{\partial f}{\partial x_{0}}, \ldots, \frac{\partial f}{\partial x_{N}}\right) \neq 0$ for at least one $i \in\{0, \ldots, N\}$. Letting $T=V(g) \subset \mathbb{P}^{N *}$ the previous condition is equivalent to $Z \nsubseteq \operatorname{Sing} T$.

Definition 1.8. Let $T=V(g) \subset \mathbb{P}^{N *}$ be an irreducible and reduced hypersurface containing the polar image $Z(f)$, where $g\left(y_{0}, \ldots, y_{N}\right)$ is as above. By definition of $g$ the variety $Z \not \subset$ Sing $T$ so that $\psi_{g}=\Phi_{g} \circ \Phi_{f}: \mathbb{P}^{N} \rightarrow \mathbb{P}^{N}$ is well defined. Equivalently $\psi_{g}$ is the composition of $\phi_{f}$ with the Gauss map of $T$. If the polynomials $g_{i}:=\frac{\partial g}{\partial y_{i}}\left(\frac{\partial f}{\partial x_{0}}, \ldots, \frac{\partial f}{\partial x_{N}}\right) \in \mathbb{K}\left[x_{0}, \ldots, x_{N}\right]$ have a common divisor $\rho:=$ g.c.d. $\left(g_{0}, \ldots, g_{N}\right) \in \mathbb{K}\left[x_{0}, \ldots, x_{N}\right]$, set $h_{i}:=\frac{g_{i}}{\rho} \in \mathbb{K}\left[x_{0}, \ldots, x_{N}\right]$, for $i=0, \ldots, N$.

It follows that the map $\psi_{g}$ is given by:

$$
\psi_{g}(p)=\left(g_{0}\left(f_{0}(p), \ldots, f_{N}(p)\right): \ldots: g_{N}\left(f_{0}(p), \ldots, f_{N}(p)\right)\right)=\left(h_{0}(p): \ldots: h_{N}(p)\right),
$$

with g.c.d. $\left(h_{0}, \ldots, h_{N}\right)=1$.

Set $T_{Z}^{*}:=\overline{\psi_{g}\left(\mathbb{P}^{N}\right)}$ and note that, by definition of $\psi_{g}, T_{Z}^{*} \subset Z(f)^{*}$. Therefore by taking $\alpha+1=\operatorname{codim}(Z)$ polynomials $g^{0}, \ldots g^{\alpha}$ defining locally $Z$ around a point $z \in Z_{\text {reg }}$ we deduce that $\left(T_{z} Z\right)^{*} \subset Z^{*}$. Moreover for a general point $r \in\left(T_{\Phi_{X}(p)} Z\right)^{*}, p \in \mathbb{P}^{N}$ such that $\Phi_{X}(p) \in Z_{\mathrm{reg}}$, there exists $a_{0}, \ldots, a_{\alpha} \in \mathbb{K}$ such that, letting $\underline{a}=\left(a_{0}, \ldots, a_{\alpha}\right) \in \mathbb{K}^{\alpha+1}$ and $g_{\underline{a}}=\sum_{i=0}^{\alpha} a_{i} g^{i}$,

$$
r=\sum_{i=0}^{\alpha} a_{i} \psi_{g^{i}}(p)=\psi_{g_{\underline{a}}}(p) .
$$

Let us recall a fundamental result proved by Gordan and Noether (see [GN] and [Lo, 2.7]).

Theorem 1.9. (Gordan-Noether Identity) Let notation be as above and let $F \in \mathbb{K}\left[x_{0}, \ldots, x_{N}\right]$. Then:

$$
\sum_{i=0}^{N} \frac{\partial F}{\partial x_{i}} h_{i}=0 \Leftrightarrow F(\mathbf{x})=F\left(\mathbf{x}+\lambda \psi_{g}(\mathbf{x})\right) \forall \lambda \in \mathbb{K} .
$$


A result used many times in the sequel is the following immediate consequence of the Gordan-Noether Identity.

Corollary 1.10. Let $X=V(f) \subset \mathbb{P}^{N}$ be a hypersurface with vanishing hessian and let notation be as above. Then

i) for every $p \in \mathbb{P}^{N} \backslash \operatorname{Sing} X$ such that $\Phi_{X}(p) \in Z_{\text {reg }}$ we have $<p,\left(T_{\Phi_{X}(p)} Z\right)^{*}>\subseteq$ $\Phi_{X}^{-1}\left(\Phi_{X}(p)\right)$;

ii) for $p \in \mathbb{P}^{N}$ general, the irreducible component of $\overline{\Phi_{X}^{-1}\left(\Phi_{X}(p)\right)}$ passing through $p$ is $<p,\left(T_{\Phi_{X}(p)} Z\right)^{*}>$. In particular for $p \in \mathbb{P}^{N}$ general $\overline{\Phi_{X}^{-1}\left(\Phi_{X}(p)\right)}$ is a union of linear spaces of dimension equal to $\operatorname{codim}(Z)$ passing through $\left(T_{\Phi_{X}(p)} Z\right)^{*}$.

iii)

$$
Z^{*} \subseteq \operatorname{Sing} X
$$

Proof. Note that

$$
\sum_{i=0}^{N} \frac{\partial^{2} f}{\partial x_{i} \partial x_{j}} h_{i}=0 \text { for every } j=0, \ldots, N .
$$

This relation is obtained by differentiating the equation $g\left(\frac{\partial f}{\partial x_{0}}, \ldots, \frac{\partial f}{\partial x_{N}}\right)=0$ with respect to $x_{j}$ and applying the chain rule. As a consequence, we get the following relation by Theorem 1.9

$$
\frac{\partial f}{\partial x_{i}}(\mathbf{x})=\frac{\partial f}{\partial x_{i}}\left(\mathbf{x}+\lambda \psi_{g}(\mathbf{x})\right) \text { for every } j=0, \ldots, N,
$$

for every $\lambda \in \mathbb{K}$ and for every $g \in \mathbb{K}\left[y_{0}, \ldots, y_{N}\right]$ such that $g\left(\frac{\partial f}{\partial x_{0}}, \ldots, \frac{\partial f}{\partial x_{N}}\right)=0$.

Let $p \in \mathbb{P}^{N} \backslash$ Sing $X$ such that $\Phi_{X}(p) \in Z_{\text {reg. }}$. Let $r \in\left(T_{\Phi_{X}(p)} Z\right)^{*}$ be a general point. By (5) we can suppose $r=\psi_{g}(p)$ so that (9) yields that the line $\langle p, r\rangle$ is contracted to the point $\Phi_{X}(p)$ and that $r=<p, r>\cap \operatorname{Sing} X$. The generality of $r$ implies that the linear space $\mathbb{P}^{\operatorname{codim}(Z)}=<p,\left(T_{\Phi_{X}(p)} Z\right)^{*}>$ is contained in $\Phi_{X}^{-1}\left(\Phi_{X}(p)\right)$, proving i) and also that $\left(T_{\Phi_{X}(p)} Z\right)^{*} \subset \operatorname{Sing} X$. Moreover since, by definition, $Z^{*}$ is ruled by the linear spaces $\left(T_{\Phi_{X}(p)} Z\right)^{*}$ part iii) immediately follows.

If $p \in \mathbb{P}^{N}$ is general, then, by generic smoothness, the irreducible component of $\Phi_{X}^{-1}\left(\Phi_{X}(p)\right)$ passing through $p$ has $\operatorname{dimension} \operatorname{codim}(Z)$ and it is smooth at $p$ so that it coincides with $<p,\left(T_{\Phi_{X}(p)} Z\right)^{*}>$, proving ii).

\section{The Perazzo map of hypersurfaces With VANishing Hessian}

Let us recall the so called Reciprocity Law of Polarity to be used later on in the analysis of the geometry of $Z^{*}$. We define first the notion of degree $s$ Polar hypersurface of $X=V(f) \subset$ $\mathbb{P}^{N}$. 
Definition 2.1. For every $s=1, \ldots, d-1$ and for every $p \in \mathbb{P}^{N}$ the degree $s$ Polar of $X$ with respect to $p$ is the hypersurface

$$
H_{p}^{s}(f):=V\left(\sum_{i_{0}+\ldots+i_{N}=s} \frac{\partial^{s} f}{\partial x_{0}^{i_{0}} \ldots x_{N}^{i_{N}}}(p) x_{0}^{i_{0}} \ldots x_{N}^{i_{N}}\right) \subset \mathbb{P}^{N} .
$$

By definition $\operatorname{deg}\left(H_{p}^{s}(f)\right)=s$ if the polynomial on the right in the above expression is not identically zero. Otherwise we naturally put $H_{p}^{s}(f)=\mathbb{P}^{N}$. For $s=1$ the hyperplane $H_{p}^{1}(f)$ is the polar hyperplane of $X$ with respect to $p$, which will be indicated simply by $H_{p}$. For $s=2$ the hypersurface $H_{p}^{2}(f)$ is a quadric hypersurface whose associated symmetric matrix is $\operatorname{Hess}_{X}(p)$ and we shall put, by abusing notation, $Q_{p}=H_{p}^{2}(f)$ if the reference to $f$ is well understood.

We recall a classical result used repeatedly in the sequel. For a proof and other applications one can consult the first chapter of [Do].

Proposition 2.2. (Reciprocity Law of Polarity) Let $X=V(f) \subset \mathbb{P}^{N}$ be a degree $d$ hypersurface. Then for every $s=1, \ldots, d-1$ and for every distinct points $p, q \in \mathbb{P}^{N}$ we have

$$
p \in H_{q}^{s}(f) \Longleftrightarrow q \in H_{p}^{d-s}(f) .
$$

In particular we have

$$
\left\{p \in X: \operatorname{mult}_{p}(X) \geq s\right\}=\bigcap_{q \in \mathbb{P}^{N}} H_{q}^{d-s+1} .
$$

Perazzo introduced in $[\mathrm{Pe}$ the notion of (Perazzo) rank of a cubic hypersurface with vanishing hessian, which we now extend to the general case. Although he does not explicitly define the rational map described below, its use was implicit in his analysis, see loc. cit.

Definition 2.3. Let $X=V(f) \subset \mathbb{P}^{N}$ be a reduced hypersurface with vanishing hessian, let $\Phi_{X}: \mathbb{P}^{N} \rightarrow \mathbb{P}^{N}$ be its polar map and let $Z=\overline{\Phi_{X}\left(\mathbb{P}^{N}\right)} \subsetneq\left(\mathbb{P}^{N}\right)^{*}$ be its polar image. The Perazzo map of $X$ is the rational map:

$$
\begin{array}{cccc}
\mathcal{P}_{X}: \mathbb{P}^{N} & \rightarrow & \mathbb{G}(\operatorname{codim}(Z)-1, N) \\
p & \mapsto & \left(T_{\Phi_{X}(p)} Z\right)^{*}
\end{array}
$$

defined as above in the open set $\mathcal{U}=\Phi_{X}^{-1}\left(Z_{\text {reg }}\right)$, where $Z_{\text {reg }}$ is the locus of smooth points of $Z$.

With this notation we have that for $p \in \mathbb{P}^{N}$ general point $\operatorname{Sing} Q_{p}=\left(T_{\Phi_{X}(p)} Z\right)^{*}$. Moreover by definition $\mathcal{P}_{X}$ is the composition of $\Phi_{X}$ with the dual Gauss map of $Z$ introduced in Definition 1.7.

The image of the Perazzo map will be denoted by $W_{X}=\overline{\mathcal{P}_{X}\left(\mathbb{P}^{N}\right)} \subset \mathbb{G}(\operatorname{codim}(Z)-1, N)$, or simply by $W$, and $\mu=\operatorname{dim} W$ is called the Perazzo rank of $X$.

We shall always identify $\left(\mathbb{P}^{N}\right)^{* *}$ with the original $\mathbb{P}^{N}$ so that, if $\operatorname{codim}(Z)=1$, we also use the identification $\mathbb{G}(0, N)=\mathbb{P}^{N}$. Let us remark that by definition and with the previous identifications we have

$$
Z^{*}=\overline{\bigcup_{z \in Z_{\mathrm{reg}}}\left(T_{z} Z\right)^{*}} \subset \mathbb{P}^{N}
$$


Remark 2.4. If $\mu=0$, then $Z \subset\left(\mathbb{P}^{N}\right)^{*}$ is a linear space and $X$ is a cone such that $\operatorname{Vert}(X)=Z^{*}=\mathbb{P}^{\operatorname{codim}(Z)-1} \subseteq X$.

Therefore if $X=V(f) \subset \mathbb{P}^{N}$ is a hypersurface with vanishing hessian, not a cone, then $\mu \geq 1$.

If $\operatorname{codim}(Z)=1$ and if $Z=V(g)$, then $\mathcal{P}_{X}=\psi_{g}$, where $\psi_{g}$ is the rational map introduced in Definition [1.8, and $W=Z^{*}$.

The following result will be useful to determine the structure of particular classes of cubic hypersurfaces with vanishing hessian defined in the sequel. First let us remark that for a cubic hypersurface and for $r, s \in \mathbb{P}^{N}$ we have: $r \in \operatorname{Sing} Q_{s}$ if and only if $s \in \operatorname{Sing} Q_{r}$.

Theorem 2.5. Let $X=V(f) \subset \mathbb{P}^{N}$ be a cubic hypersurface with vanishing hessian. Let $w=\left[\left(T_{\Phi_{X}(p)} Z\right)^{*}\right] \in W_{X} \subset \mathbb{G}(\operatorname{codim}(Z)-1, N)$ be a general point and let $r \in\left(T_{\Phi_{X}(p)} Z\right)^{*}$ be a general point with $p \in \mathbb{P}^{N}$ general. Then:

$$
\overline{\mathcal{P}_{X}^{-1}(w)}=\bigcap_{r \in\left(T_{\Phi_{X}(p)} Z\right)^{*}=\operatorname{Sing} Q_{p}} \operatorname{Sing} Q_{r}=\mathbb{P}_{w}^{N-\mu} .
$$

Proof. By definition $\overline{\mathcal{P}_{X}^{-1}(w)}$ is the closure of the set of all (general) $p^{\prime} \in \mathbb{P}^{N}$ such that $\operatorname{Sing}\left(Q_{p^{\prime}}\right)=\operatorname{Sing}\left(Q_{p}\right)$. This happens if and only if $r \in \operatorname{Sing}\left(Q_{p}\right)$ implies $r \in \operatorname{Sing}\left(Q_{p^{\prime}}\right)$ (or vice versa by symmetry and by the generality of $\left.p, p^{\prime} \in \mathbb{P}^{N}\right)$, which in turn happens if and only if $p^{\prime} \in \operatorname{Sing}\left(Q_{r}\right)$ for every $r \in \operatorname{Sing} Q_{p}$, yielding the first equality in (12) and concluding the proof.

Remark 2.6. $\quad$ i) In [Pe, $\S 5$, pg. 339] Perazzo states that $\overline{\mathcal{P}_{X}^{-1}(w)}$ is a linear space of dimension $N-\mu$ but in our opinion his arguments contain some gaps.

Indeed, if $\operatorname{codim}(Z)=1,\left(T_{\Phi_{X}(p)} Z\right)^{*}=r$ is a point and (12) gives $\overline{\mathcal{P}_{X}^{-1}(w)}=$ Sing $Q_{r}$. Perazzo wrongly claims that $\operatorname{Sing} Q_{r}=\overline{\mathcal{P}_{X}^{-1}(w)}$ for $r \in\left(T_{\Phi_{X}(p)} Z\right)^{*}$ general also when $\operatorname{dim}\left(\left(T_{\Phi_{X}(p)} Z\right)^{*}\right)=\operatorname{codim}(Z)-1>0$. To prove this wrong claim Perazzo erroneously assumes that the tangent space $T_{r} Z^{*}$ is constant for $r \in\left(T_{\Phi_{X}(p)} Z\right)^{*}$ general when $\left.\operatorname{dim}\left(T_{\Phi_{X}(p)} Z\right)^{*}\right)>0$. This claim would be equivalent to $\operatorname{dim}\left(Z^{*}\right)=$ $\operatorname{codim}(Z)-1+\mu$ as we shall see in Proposition 2.7 below, a fact used by Perazzo repeatedly in his paper.

Perazzo probably confuses the contact locus of a general tangent space to $Z^{*}$, that is the closure of a general fiber of the Gauss map of $Z^{*}$, with the contact locus of a general hyperplane tangent to $Z^{*}$, which is a linear space of the form $\left(T_{\Phi_{X}(p)} Z\right)^{*}$. For $\operatorname{codim}(Z)>1$ these contact loci can differ and the linear spaces $\operatorname{Sing} Q_{r}$ can depend on $r \in\left(T_{\Phi_{X}(p)} Z\right)^{*}$.

ii) Formula (12) is a particular case of general results on linear systems of quadrics with tangential defect, see [De, [La] and particularly [Al, Corollary 1] where a proof of (12) is deduced from these general facts. As shown by the examples in the last pages of [Al] the fibers of the Perazzo map are not necessarily linear for hypersurfaces of 
degree greater than three. The general structure of the fibers of the Perazzo map for arbitrary hypersurfaces is, to the best of our knowledge, unknown.

In order to better clarify the wrong claims made by Perazzo and outlined in part i) of the previous Remark we collect some facts which will be also used later.

Proposition 2.7. Let notation be as above and let $F=\mathbb{P}^{\operatorname{dim}(Z)-\mu} \subset Z$ be the closure of the fiber of the Gauss map of $Z$ passing through the general point $\Phi_{X}(p) \in Z$. Then:

a) $\operatorname{dim}\left(Z^{*}\right) \leq \operatorname{codim}(Z)-1+\mu$;

b) if $r \in\left(T_{\Phi_{X}(p)} Z\right)^{*} \subset Z^{*}$ is a general point, then

$$
F \subseteq\left(T_{r} Z^{*}\right)^{*}
$$

with equality if and only if $\operatorname{dim}\left(Z^{*}\right)=\operatorname{codim}(Z)-1+\mu$.

c) If $\operatorname{codim}(Z)=1$, then $F^{*}=T_{r} Z^{*}$.

d) If $w=\left[\left(T_{\Phi_{X}(p)} Z\right)^{*}\right]$, then

$$
\bigcap_{q \in \mathbb{P}_{w}^{N-\mu}} H_{q}=F^{*} \supseteq T_{r} Z^{*}
$$

Proof. Let us remark that (11) yields $\operatorname{dim}\left(Z^{*}\right) \leq \operatorname{codim}(Z)-1+\mu$ with equality if and only if through a general point $r \in Z^{*}$ there passes a finite number of linear spaces of the form $\left(T_{\Phi_{X}(p)} Z\right)^{*}$, proving a).

Let $r \in\left(T_{\Phi_{X}(p)} Z\right)^{*} \subset Z^{*}$ be a general point. By Reflexivity $\left(T_{r} Z^{*}\right)^{*}$ is the contact locus on $Z$ of the hyperplane $r^{*} \in Z$, tangent to $Z$ at the point $\Phi_{X}(p)$ (to deduce this one remarks that $\left.r \in\left(T_{\Phi_{X}(p)} Z\right)^{*}\right)$. Thus, since $F$ is the contact locus on $X$ of $T_{\Phi_{X}(p)} Z$, we get

$$
F \subseteq\left(T_{r} Z^{*}\right)^{*}
$$

with equality if and only if $\operatorname{dim}\left(Z^{*}\right)=\operatorname{codim}(Z)-1+\mu$. Furthermore if $\operatorname{codim}(Z)=1$, then $F^{*}=T_{r} Z^{*}$ and the proofs of b) and c) are complete.

Moreover, if $\operatorname{dim}\left(Z^{*}\right)=\operatorname{codim}(Z)-1+\mu$, we claim that there exists a unique linear space of the form $\left(T_{\Phi_{X}(p)} Z\right)^{*}$ passing through a general point of $Z^{*}$. Indeed since $F^{*}=T_{r} Z^{*}, T_{r} Z^{*}$ is independent of $r \in\left(T_{\Phi_{X}(p)} Z\right)^{*}$. Therefore $\left(T_{\Phi_{X}(p)} Z\right)^{*}$ is contained in the contact locus on $Z^{*}$ of the general tangent space $T_{r} Z^{*}$ from which it follows that $\left(T_{\Phi_{X}(p)} Z\right)^{*}$ is the (closure of the) fiber of the Gauss map of $Z^{*}$ passing through $r$, proving the claim.

If $H$ is a general hyperplane such that $H \supseteq F^{*}$, then $[H] \in\left(F^{*}\right)^{*}=F \subset Z$ so that $[H]=\Phi_{X}(q)$ with $q \in \mathbb{P}_{w}^{N-\mu}$ and $H=H_{q}$. Thus

$$
\bigcap_{q \in \mathbb{P}_{w}^{N-\mu}} H_{q}=F^{*} \supseteq T_{r} Z^{*},
$$

proving $d)$. 
Theorem 2.8. Let $X=V(f) \subset \mathbb{P}^{N}$ be an irreducible cubic hypersurface with vanishing hessian, not a cone, with $\operatorname{codim}(Z)=1$. Let $r=\left[\left(T_{\Phi_{X}(p)} Z\right)^{*}\right] \in W=Z^{*} \subset \mathbb{P}^{N}$ with $p \in \mathbb{P}^{N}$ general and let $X_{r}=\overline{\mathcal{P}_{X}^{-1}(r)} \cap X$, which is equidimensional of dimension $N-\operatorname{dim}\left(Z^{*}\right)-1$. Then:

$$
\operatorname{dim}\left(Z^{*}\right) \leq \frac{N-1}{2}
$$

Proof. Let us consider the cubic hypersurface $X_{r}=\mathbb{P}_{r}^{N-\operatorname{dim}\left(Z^{*}\right)} \cap X$, which could also have some non reduced component.

Let $\tilde{H}_{p}$ be the polar hyperplane of a point $p \in \mathbb{P}_{r}^{N-\operatorname{dim}\left(Z^{*}\right)}$ with respect to $X_{r}$. One immediately sees that $\tilde{H}_{p}=H_{p} \cap \mathbb{P}_{r}^{N-\operatorname{dim}\left(Z^{*}\right)}$. Since $\operatorname{codim}(Z)=1$, letting $F$ be the closure of the fiber of the Gauss map of $Z$ passing through $\Phi_{X}(p)$, we have $F^{*}=T_{r} Z^{*}$ by part b) of Proposition 2.7 so that by (14)

$$
\bigcap_{p \in \mathbb{P}_{r}^{N-\operatorname{dim}\left(Z^{*}\right)}} H_{p}=T_{r} Z^{*}
$$

Moreover

$$
T_{r} Z^{*} \subseteq T_{r} \operatorname{Sing} X=\operatorname{Sing} Q_{r}=\mathbb{P}_{r}^{N-\operatorname{dim}\left(Z^{*}\right)},
$$

where the first inclusion follows from $Z^{*} \subseteq \operatorname{Sing} X$, the first equality from definition of the scheme Sing $X$ and the last equality from (12) applied to $W=Z^{*}$.

Therefore

$$
\bigcap_{p \in \mathbb{P}_{r}^{N-\operatorname{dim}\left(Z^{*}\right)}} \tilde{H}_{p}=\left(\bigcap_{p \in \mathbb{P}_{r}^{N-\operatorname{dim}\left(Z^{*}\right)}} H_{p}\right) \cap \mathbb{P}_{w}^{N-\operatorname{dim}\left(Z^{*}\right)}=T_{r} Z^{*} \cap \mathbb{P}_{r}^{N-\operatorname{dim}\left(Z^{*}\right)}=T_{r} Z^{*}
$$

By Proposition 2.2 the linear space $T_{r} Z^{*}$ is exactly the locus of points of multiplicity three of the cubic hypersurface $X_{r} \subset \mathbb{P}_{r}^{N-\operatorname{dim}\left(Z^{*}\right)}$. Thus the cubic hypersurface $X_{r} \subset \mathbb{P}^{N-\operatorname{dim}\left(Z^{*}\right)}$ is a cone whose vertex is $T_{r} Z^{*}$. In particular $\operatorname{dim}\left(Z^{*}\right)=\operatorname{dim}\left(T_{r} Z^{*}\right) \leq N-\operatorname{dim}\left(Z^{*}\right)-1$, proving (15).

The linearity of the fibers has strong consequences on the geometry of a cubic hypersurface with vanishing hessian. Let us recall some easy and well known facts on congruences of order one of linear spaces, that is irreducible families $\Theta \subset \mathbb{G}(\beta, N)$ of linear spaces of dimension $\beta>0$ such that through a general point of $\mathbb{P}^{N}$ there passes a unique member of the family. Let us remark that the previous condition forces $\operatorname{dim}(\Theta)=N-\beta$ and that the tautological map $p: \mathcal{U} \rightarrow \mathbb{P}^{N}$ from the universal family $\pi: \mathcal{U} \rightarrow \Theta$ is birational onto $\mathbb{P}^{N}$.

Let notation be as above and let

$$
V=\left\{q \in \mathbb{P}^{N} ; \#\left(p^{-1}(q)\right) \geq 2\right\}=\left\{q \in \mathbb{P}^{N} ; \operatorname{dim}\left(p^{-1}(q)\right)>0\right\} \subset \mathbb{P}^{N}
$$

be the so called jump (or branch) locus of $\Theta$.

The easiest examples of congruences of linear spaces of dimension $\beta$ is given by the family of linear spaces of dimension $\beta+1$ passing through a fixed linear space $L=\mathbb{P}^{b} e t a \subset \mathbb{P}^{N}$.

The previous examples motivate the following definition. 
Definition 2.9. An irreducible cubic hypersurface $X \subset \mathbb{P}^{N}$ with vanishing hessian, not a cone, will be called a Special Perazzo Cubic Hypersurface if the general fibers of its Perazzo map form a congruence of linear spaces passing through a fixed $\mathbb{P}^{N-\mu-1}$.

Special Perazzo Cubic Hypersufaces will be treated in Section 3 following very closely the original treatment of Perazzo and providing canonical forms and explicit geometrical descriptions in any dimension. Let us remark that for Special Perazzo Cubic Hypersurfaces the linear span of two general fibers is a $\mathbb{P}^{N-\mu+1}$ which is strictly contained in $\mathbb{P}^{N}$ if $\mu>1$. For $\mu=1$ the general fibers determine a pencil of hyperplanes and this case will be treated in arbitrary dimension in Theorem 4.1 .

To ensure that a cubic hypersuface is a Special Perazzo Cubic Hypersurface it is sufficient to control the linear span (or equivalently the intersection) of two general fibers. Indeed we have the following easy result, whose proof is left to the reader.

Lemma 2.10. Let $X=V(f) \subset \mathbb{P}^{N}$ be an irreducible cubic hypersurface with vanishing hessian, not a cone. Let $w_{1}, w_{2} \in W$ be general points, let $\overline{\mathcal{P}_{X}^{-1}\left(w_{i}\right)}=\mathbb{P}_{w_{i}}^{N-\mu}$ be the corresponding fibers of the Perazzo map. Suppose that $\mathbb{P}_{w_{1}}^{N-\mu} \cap \mathbb{P}_{w_{2}}^{N-\mu}=\mathbb{P}^{N-\mu-1}$.

Then $X \subset \mathbb{P}^{N}$ is a Special Perazzo Cubic Hypersurface.

The linear span of two general fibers $\mathbb{P}_{w_{1}}^{N-\mu}$ and $\mathbb{P}_{w_{2}}^{N-\mu}$ of the Perazzo map is strongly related to the dimension of the secant variety $S Z^{*} \subseteq \mathbb{P}^{N}$ of $Z^{*}$ at least for $\operatorname{codim}(Z)=1$. For cubic hypersurfaces with vanishing hessian we have an obvious inclusion $S Z^{*} \subseteq X$ since $Z^{*} \subseteq \operatorname{Sing} X$.

The following result, surely well known (see for example [Seg3]), has many applications in this context. The proof can be left to the reader.

Lemma 2.11. Let $\left\{Q_{\lambda}\right\}_{\lambda \in \mathbb{P}^{m}}$ be a linear system of quadric hypersurfaces in $\mathbb{P}^{N}$. Then

$$
<\overline{\bigcup_{\lambda \in \mathbb{P}^{m}} \operatorname{Sing} Q_{\lambda}}>\subset Q_{t}
$$

for $t \in \mathbb{P}^{m}$ general.

The next Proposition was inspired by [Pe, footnote pg. 348,349] and it relies essentially on the previous geometrical fact.

Proposition 2.12. Let $X=V(f) \subset \mathbb{P}^{N}$ be an irreducible cubic hypersurface with vanishing hessian, not a cone. Then the following conditions are equivalent:

i) $S Z^{*} \subseteq \operatorname{Sing} X$;

ii) $Z^{*} \subseteq \bigcap_{w \in W \text { general }} \mathbb{P}_{w}^{N-\mu}$;

iii) $\left\langle Z^{*}>\subseteq \bigcap_{w \in W \text { general }} \mathbb{P}_{w}^{N-\mu}\right.$;

iv) $<Z^{*}>\subseteq$ Sing $X$. 
Proof. Let us suppose that $S Z^{*} \subseteq \operatorname{Sing} X$. If $r_{1}, r_{2} \in Z^{*}$ are general points, then $<r_{1}, r_{2}>\subseteq$ Sing $X$ implies $r_{2} \in \operatorname{Sing} Q_{\epsilon}$ for every $\epsilon \in<r_{1}, r_{2}>$. Taking $r_{1}$ general, $r_{1} \in\left(T_{z} Z\right)^{*}$ where $w=\left[\left(T_{z} Z\right)^{*}\right] \in W$ is general, then $r_{2} \in \operatorname{Sing} Q_{r_{1}}$, which by the generality of $r_{1} \in\left(T_{z} Z\right)^{*}$ yields

$$
r_{2} \in \bigcap_{r_{1} \in\left(T_{z} Z\right)^{*}} \operatorname{Sing} Q_{r_{1}}=\mathbb{P}_{w}^{N-\mu} .
$$

By the generality of $r_{2} \in Z^{*}$ and of $w \in W$ we deduce:

$$
Z^{*} \subseteq \bigcap_{w \in W} \mathbb{P}_{w}^{N-\mu}
$$

Of course ii) is equivalent to iii).

Let us suppose now $<Z^{*}>\subseteq \bigcap_{w \in W \text { general }} \mathbb{P}_{w}^{N-\mu}$ and let $s \in<Z^{*}>$ be a general point. By hypothesis and by (12) we have that $s \in \operatorname{Sing} Q_{r}$ with $r \in Z^{*}$ general. We claim that

$$
S^{k} Z^{*} \subseteq \operatorname{Sing} X \Rightarrow S^{k+1} Z^{*} \subseteq \operatorname{Sing} X
$$

from which iv) will follow by induction.

Since $S^{k+1} Z^{*}=S\left(S^{k} Z^{*}, Z^{*}\right)$, we take $q \in S^{k} Z^{*} \subset \operatorname{Sing} X$ and $r \in Z^{*} \subset \operatorname{Sing} X$ general points. Then $q \in<Z^{*}>$ implies $q \in \operatorname{Sing} Q_{r}$ so that $\frac{\partial f}{\partial x_{i}}(\lambda q+\mu r)=\frac{\partial f}{\partial x_{i}}(q) \lambda^{2}+\frac{\partial f}{\partial x_{i}}(r) \mu^{2} \equiv 0$ since $q, r \in \operatorname{Sing} X$. Therefore $\lambda q+\mu r \in \operatorname{Sing} X$, which by the generality of $q$ and $r$ yields $S^{k+1} Z^{*} \subset \operatorname{Sing} X$, as claimed.

The last implication iv) $\Rightarrow \mathrm{i}$ ) is obvious.

Example 1. Consider the Segre variety $Y=\operatorname{Seg}(1,2)=\mathbb{P}^{1} \times \mathbb{P}^{2} \subset \mathbb{P}^{5}=\mathbb{P}\left(\mathbb{M}_{2 \times 3}(\mathbb{K})\right)$. With the last identification we have

$$
\operatorname{Seg}(1,2)=\{[A] \mid \operatorname{rk}(A)=1\}
$$

and $S Y=\mathbb{P}^{5}$.

The projection of $Y \subset \mathbb{P}^{5}$ from a point $p \in \mathbb{P}^{5} \backslash Y$ is a cubic hypersurface $X=V(f) \subset \mathbb{P}^{4}$. It is easy to check that $(\operatorname{Sing} X)_{\text {red }}=\mathbb{P}^{2}$ because the secant lines to $Y$ passing through $p$ describe a two dimensional quadric surface $\Sigma_{p} \subset Y$.

We claim that $X$ has vanishing hessian. Indeed, by the Reciprocity Law of Polarity, the polar quadric $Q_{p}$ of a general point $p \in \mathbb{P}^{4}$ is a cone being a 3-dimensional quadric containing the plane $(\operatorname{Sing} X)_{\text {red }}$, see also Proposition 3.1 for generalizations of this argument. Furthermore $X$ is not a cone since its dual is clearly non degenerate being a general hyperplane section of $Y^{*} \simeq Y$. The example recalled in the Introduction is projectively equivalent to $X$. Indeed, modulo projective equivalence, $V\left(x_{0} x_{3}^{2}+x_{1} x_{3} x_{4}+x_{2} x_{4}^{2}\right) \subset \mathbb{P}^{4}$ is the only cubic hypersurface in $\mathbb{P}^{4}$ with vanishing hessian and not a cone, see Theorem 4.2 .

Since $Z^{*} \subset(\operatorname{Sing} X)_{\text {red }}=\mathbb{P}^{2}=\Pi, Z \subset \mathbb{P}^{4}$ is a cone over the dual curve of $Z^{*}$ as a plane curve and whose vertex is the line $L=\Pi^{*}$. Thus $\operatorname{codim}(Z)=1$ and $\mu=1$. One easily deduces that $X \subset \mathbb{P}^{4}$ is a Special Perazzo Cubic Hypersurface whose associated congruence is given by the family of $\mathbb{P}^{3}$ 's passing through $(\operatorname{Sing} X)_{\text {red }}$, see also Proposition 2.12 . 
Proposition 2.13. Let $X=V(f) \subset \mathbb{P}^{N}$ be an irreducible cubic hypersurface with vanishing hessian, not a cone, with $\operatorname{codim}(Z)=1$. Let $w_{1}, w_{2} \in W=Z^{*}$ be general points and let $\overline{\mathcal{P}_{X}^{-1}\left(w_{i}\right)}=\mathbb{P}_{w_{i}}^{N-\mu}$ be the corresponding fibers of the Perazzo map. If $\left\langle\mathbb{P}_{w_{1}}^{N-\mu}, \mathbb{P}_{w_{2}}^{N-\mu}\right\rangle=\mathbb{P}^{N}$, then $<Z^{*}>\subseteq$ Sing $X$.

Proof. Let $p \in \mathbb{P}^{N}$ be a general point. Then there exist general $p_{i} \in \mathbb{P}_{w_{i}}^{N-\mu}$ such that $p \in<$ $p_{1}, p_{2}>$. Since $w_{i}=\mathcal{P}_{X}\left(p_{i}\right)=\operatorname{Sing} Q_{p_{i}}$, Lemma 2.11 implies $<Z^{*}>\subseteq Q_{p}$ for $p \in \mathbb{P}^{N}$ general. To conclude it suffices to recall that

$$
(\operatorname{Sing} X)_{\mathrm{red}}=\bigcap_{p \in \mathbb{P}^{N} \text { general }} Q_{p}
$$

by Proposition 2.2

When $\operatorname{codim}(Z)=1$ there are interesting relations between the base locus of the Perazzo map and $W=Z^{*}$. Indeed if $Z=V(g)$, letting notation be as in Definition 1.8, we have

$$
\mathcal{P}_{X}=\psi_{g}=\left(h_{0}: \ldots: h_{N}\right): \mathbb{P}^{N} \rightarrow \mathbb{P}^{N}
$$

and $Z^{*}=W=\overline{\psi_{g}\left(\mathbb{P}^{n}\right)}$.

Proposition 2.14. Let $X=V(f) \subset \mathbb{P}^{N}$ be an irreducible cubic hypersurface with vanishing hessian, not a cone, with $\operatorname{codim}(Z)=1$ and let $\operatorname{Bs}\left(\mathcal{P}_{X}\right)=\operatorname{Bs}\left(\psi_{g}\right)=V\left(h_{0}, \ldots, h_{N}\right)$ be the base locus scheme of $\mathcal{P}_{X}$. Then:

a) $Z^{*} \subseteq \operatorname{Bs}\left(\mathcal{P}_{X}\right)$;

b) if $X$ is a Special Perazzo Cubic Hypersurface, then

$$
Z^{*} \subseteq \bigcap_{w \in W \text { general }} \mathbb{P}_{w}^{N-\mu}=\mathbb{P}^{N-\mu-1} \subseteq \operatorname{Bs}\left(\mathcal{P}_{X}\right)
$$

Proof. Let notation be as in Subsection 1.2 with $Z=V(g) \subset \mathbb{P}^{N *}$. Since $g_{i}=\rho \cdot h_{i}$ for every $i=0, \ldots, N$ and since $g_{i}\left(\mathbf{x}+\lambda \psi_{g}(\mathbf{x})\right)=g_{i}(\mathbf{x})$ for every $i=0, \ldots, N$ and for every $\lambda \in \mathbb{K}$, we deduce $h_{i}\left(\mathbf{x}+\lambda \psi_{g}(\mathbf{x})\right)=h_{i}(\mathbf{x})$ for every $i=0, \ldots, N$ and for every $\lambda \in \mathbb{K}$. Thus

$$
\psi_{g}\left(\mathbf{x}+\lambda \psi_{g}(\mathbf{x})\right)=\psi_{g}(\mathbf{x})
$$

for every $\lambda \in \mathbb{K}$. In particular we have $h_{i}\left(\psi_{g}(p)\right)=0$ for every $i=0, \ldots, N$, proving a).

To prove b) let us remark that $\mathbb{P}_{w}^{N-\mu} \cap \operatorname{Bs}\left(\mathcal{P}_{X}\right)$ is a hypersurface of degree $e=\operatorname{deg}\left(h_{i}\right) \geq 1$ and that, by hypothesis, the intersection of two general $\mathbb{P}_{w_{i}}^{N-\mu}$, s is a $\mathbb{P}^{N-\mu-1}=L$ contained in $\operatorname{Bs}\left(\mathcal{P}_{X}\right)$ and in a general $\mathbb{P}_{w}^{N-\mu}$. Thus $L$ is an irreducible component of $\mathbb{P}_{w}^{N-\mu} \cap \operatorname{Bs}\left(\mathcal{P}_{X}\right)$. Let $p \in \mathbb{P}_{w}^{N-\mu}$ be a general point. By (19) the line $<p, \psi_{g}(p)>$, which is contained in $\mathbb{P}^{N-\mu}$, cuts $\operatorname{Bs}\left(\mathcal{P}_{X}\right)$ only in the point $\psi_{g}(p)$, yielding $\psi_{g}(p) \in L$ because $<p, \psi_{g}(p)>\cap L \neq \emptyset$. The generality of $w$ implies $\psi_{g}(p) \in L$ for $p \in \mathbb{P}^{N}$ general so that $Z^{*} \subseteq L$. 


\section{Classes of Cubic hypersurfaces with vanishing hessian according to Perazzo and canonical forms of Special Perazzo Cubic Hypersurfaces}

The examples of hypersurfaces with vanishing hessian not cones considered in GN and in Perazzo $\mathrm{Pe}$ are singular along linear spaces, see also [CRS]. We now prove that in some cases the existence of a linear space of sufficiently high dimension in the singular locus assures that a cubic hypersurface has vanishing hessian.

Proposition 3.1. Let $X \subset \mathbb{P}^{N}, N \geq 4$, be an irreducible cubic hypersurface. Suppose that there exists a linear space $S=V\left(x_{\tau+1}, \ldots, x_{N}\right)=\mathbb{P}^{\tau}$ contained in the singular locus of $X$. Then, up to projective transformations:

$$
f=\sum_{i=0}^{\tau} x_{i} C^{i}\left(x_{\tau+1}, \ldots, x_{N}\right)+D\left(x_{\tau+1}, \ldots, x_{N}\right),
$$

with $C^{i}\left(x_{\tau+1}, \ldots, x_{N}\right)$ a quadratic form for every $i=0, \ldots, \tau$ and with $D\left(x_{\tau+1}, \ldots, x_{N}\right)$ a cubic form.

i) If $\tau>\frac{N-1}{2}$, then $\operatorname{hess}_{X}=0$;

ii) If $\tau>\frac{(N-\tau)(N-\tau+1)}{2}-1$, then $X$ is a cone.

In particular, for $N>4$ every non normal cubic hypersurface is a cone.

Proof. The proof of (20) is left to the reader. To prove i) it is enough to remark that $S \subseteq \operatorname{Sing} X \subset Q_{p}$ with $p \in \mathbb{P}^{N}$ general by the Reciprocity Law of Polarity. If $\tau>\frac{N-1}{2}$, then $Q_{p}$ is a cone and the generality of $p$ implies hess $X=0$.

To prove ii) we observe that $f_{i}=C_{i}$ for every $i=0, \ldots, \tau$ and that the number of independent quadrics in $N-\tau$ variables is $\left(\begin{array}{c}N-\tau+1 \\ 2\end{array}\right)$.

The cubic hypersurface is non normal if and only if to $\operatorname{dim}(\operatorname{Sing} X)=N-2$, which on the other hand implies $(\operatorname{Sing} X)_{\text {red }}=\mathbb{P}^{N-2}$. Therefore we can assume $N>4$ and $\tau=N-2$ so that $X$ is a cone by part ii).

Remark 3.2. For cubic hypersurfaces in $\mathbb{P}^{5}$ the existence of a singular plane does not imply the vanishing of the hessian. Indeed, if

$$
X=V\left(x_{0} x_{3}^{2}+x_{1} x_{4}^{2}+x_{2} x_{5}^{2}\right) \subset \mathbb{P}^{5}
$$

then $(\operatorname{Sing} X)_{\text {red }}=V\left(x_{3}, x_{4}, x_{5}\right)=\mathbb{P}^{2}$ but hess $X \neq 0$. Thus the bound in Proposition 3.1 is sharp for any dimension by easy manipulations on the previous example.

Via direct computations one can verify that for a general $p \in \mathbb{P}^{5}$ the non-singular quadric $Q_{p}$ contains the linear space $S=V\left(x_{3}, x_{4}, x_{5}\right)$ and that $\cap_{s \in S} T_{s} Q_{p}=S$.

We shall now introduce some terminology in order to obtain a projective characterization of Special Perazzo Cubic Hypersurfaces.

This approach follows strictly the original work of Perazzo, who used the next results to provide simplified canonical forms for Special Perazzo Cubic Hypersurfaces, see [Pe, §14-18 p. 344-350].

The first definition is a generalization of the classical notion of generatrix of a cone with vertex a point, that is a line contained in the cone and passing through the vertex. 
Definition 3.3. Let $Q \subset \mathbb{P}^{N}$ be a a quadric of $\operatorname{rank} \operatorname{rk}(Q)=\beta \geq 3$ and let $\operatorname{Vert}(Q)=$ Sing $Q=\mathbb{P}^{N-\beta}$ be its vertex. A linear space $M=\mathbb{P}^{\tau}, \tau \geq 1$, is a generator of $Q$ if

$$
\operatorname{Vert}(Q) \subsetneq M \subset Q \text {. }
$$

The proof of the next result is left to the reader.

Lemma 3.4. Let $Q \subset \mathbb{P}^{N}$ be a quadric of rank $\operatorname{rk}(Q)=\beta$ and let $M=\mathbb{P}^{\tau}$ be a generator of Q. Then

$$
\bigcap_{m \in M \backslash \operatorname{Vert}(Q)} T_{m} Q=\mathbb{P}\left(\Phi_{Q}(M)\right)^{*}=\mathbb{P}^{2 N-\beta-\tau} .
$$

Definition 3.5. Let $Q \subset \mathbb{P}^{N}$ be a quadric, let $M$ be a generator of $Q$ and let $L$ be a linear space containing $M$. The quadric is tangent to the linear space $L$ along the generator $M$ if $L \subseteq T_{m} Q$ for all $m \in M$.

Obviously a necessary condition for $L$ being tangent to $Q$ along $M=\mathbb{P}^{\tau}$ is

$$
L \subseteq \bigcap_{m \in M \backslash \operatorname{Vert}(Q)} T_{m} Q=\mathbb{P}^{2 N-\mathrm{rk}(Q)-\tau},
$$

yielding $\operatorname{dim}(M)=\tau \leq \operatorname{dim}(L) \leq 2 N-\operatorname{rk}(Q)-\tau$.

In the example analyzed in Remark 3.2 the general polar quadric $Q_{p} \subset \mathbb{P}^{5}$, which is nonsingular, is tangent to $M=V\left(x_{3}, x_{4}, x_{5}\right)=(\operatorname{Sing} X)_{\text {red }}$ along $M$.

The next result is the core of the work of Perazzo on canonical forms. We shall always assume $\operatorname{codim}(Z)=1$ or equivalently $\operatorname{rk}\left(Q_{p}\right)=N$ for $p \in \mathbb{P}^{N}$ general which corresponds to the case $p=0$ considered by Perazzo. We shall obtain a simplified canonical form refining Proposition 3.1, corresponding to the case in which the polar quadric of a general point of $\mathbb{P}^{N}$ is tangent to a fixed linear space $\mathbb{P}^{N-\tau}$ along a common generator of dimension $\tau$, see [Pe, $\S 1.17$ p.349].

We shall consider Sing $Q_{p} \subset M \subset Q_{p}$ with $M=\mathbb{P}^{\tau}$ a common generator having equations: $x_{\tau+1}=\ldots=x_{N}=0$. Furthermore we shall suppose

$$
L=\bigcap_{m \in M \backslash \operatorname{Vert}\left(Q_{p}\right)} T_{m} Q_{p}=\mathbb{P}^{N-\tau},
$$

that is $L$ will always be the maximal linear subspace containing $M$ along which the quadric $Q_{p}$ can be tangent along the generator $M$. Moreover we shall assume that $L$ has equations: $x_{N-\tau+1}=\ldots=x_{N}=0$. Under these hypothesis $\operatorname{dim}(L)=N-\tau \geq \tau=\operatorname{dim}(M)$ with equality if and only if $L=M$.

Theorem 3.6. Let $X=V(f) \subset \mathbb{P}^{N}$ be a cubic hypersurface having vanishing hessian, not a cone, and with $\operatorname{codim}(Z)=1$. Suppose that the polar quadric of a general point of $\mathbb{P}^{N}$ is tangent to a fixed linear space $L=\mathbb{P}^{N-\tau}$ along a common generator $M$ with $M=$ $V\left(x_{\tau+1}, \ldots, x_{N}\right)$ and $L=V\left(x_{N-\tau+1}, \ldots, x_{N}\right)$. Then:

$$
f=\sum_{i=0}^{\tau} x_{i} C^{i}\left(x_{N-\tau+1}, \ldots, x_{N}\right)+D\left(x_{\tau+1}, \ldots, x_{N}\right),
$$


where the $C_{i}$ 's are linearly independent quadratic forms depending only on the variables $x_{N-\tau+1}, \ldots, x_{N}$ and $D$ is a cubic in the variables $x_{\tau+1}, \ldots, x_{N}$.

On the contrary, for every cubic hypersurface $X=V(f) \subset \mathbb{P}^{N}$ with $f$ as in (21) the polar quadric of a general point of $\mathbb{P}^{N}$ is tangent to $V\left(x_{N-\tau+1}, \ldots, x_{N}\right)$ along the common generator $V\left(x_{\tau+1}, \ldots, x_{N}\right)$. Moreover such a cubic hypersurface has vanishing hessian and $\operatorname{codim}(Z)=1$.

Proof. For notational simplicity in the formulas let $m=N-\tau$. Let $s=\left(b_{0}: \ldots: b_{\tau}: 0: \ldots\right.$ : $0) \in M$ and let $q \in L \subset T_{s} Q_{p}, q=\left(a_{0}: \ldots: a_{\tau}: a_{\tau+1}: \ldots: a_{m}: 0: \ldots: 0\right)$.

The condition $q \in T_{s} Q_{p}$ for every $q \in L$ and for every $s \in M$ is equivalent to $s Q_{p} q^{t}=0$ for all $q \in L$ and for all $s \in \mathbb{P}^{\tau}$, where by abusing notation we identify $Q_{p}$ with its associated symmetric matrix.

Using the canonical form of Proposition 3.1, we obtain $\frac{\partial f}{\partial x_{i}}=C^{i} i=0, \ldots, \tau$ and $\frac{\partial f}{\partial x_{i}}=$ $\sum_{j=0}^{\tau} x_{j} C_{i}^{j}+D_{i} i=\tau+1, \ldots, N$. Hence $\frac{\partial^{2} f}{\partial x_{i} \partial x_{j}}=C_{j}^{i}$ for $i=0, \ldots, \tau$ and $j=\tau+1, \ldots, m$. In coordinates:

$$
\left[b_{0}: \ldots: b_{\tau}: 0: \ldots: 0\right]\left[\begin{array}{ccccccccc}
0 & \ldots & 0 & C_{\tau+1}^{0} & \ldots & C_{m}^{0} & * & \ldots & * \\
\vdots & \ddots & \vdots & \vdots & \ddots & \vdots & \vdots & \ddots & \vdots \\
0 & \ldots & 0 & C_{\tau+1}^{\tau} & \ldots & C_{m}^{\tau} & * & \ldots & * \\
C_{\tau+1}^{0} & \ldots & C_{\tau+1}^{\tau} & * & \ldots & * & * & \ldots & * \\
\vdots & \ddots & \vdots & \vdots & \ddots & \vdots & \vdots & \ddots & \vdots \\
C_{m}^{0} & \ldots & C_{m}^{\tau} & * & \ldots & * & * & \ldots & * \\
* & \ldots & * & * & \ldots & * & * & \ldots & * \\
\vdots & \ddots & \vdots & \vdots & \ddots & \vdots & \vdots & \ddots & \vdots \\
* & \ldots & * & * & \ldots & * & * & \ldots & *
\end{array}\right]\left[\begin{array}{c}
a_{0} \\
\vdots \\
a_{\tau} \\
a_{\tau+1} \\
\vdots \\
a_{m} \\
0 \\
\vdots \\
0
\end{array}\right]=0
$$

After the first product we get

$$
\left[0: \ldots: 0: \sum_{i=0}^{\tau} b_{i} C_{\tau+1}^{i}: \ldots: \sum_{i=0}^{\tau} b_{i} C_{m}^{i}: *: \ldots: *\right]\left[\begin{array}{c}
a_{0} \\
\vdots \\
a_{\tau} \\
a_{\tau+1} \\
\vdots \\
a_{m} \\
0 \\
\vdots \\
0
\end{array}\right]=0 .
$$

Then,

$$
\sum_{\substack{0 \leq i \leq \tau \\ \tau+1 \leq j \leq m}} a_{j} b_{i} C_{j}^{i}=0 .
$$


Hence $C_{j}^{i}=0$ for $0 \leq i \leq \tau$ and for $\tau+1 \leq j \leq m$ because $a_{i}, b_{j} \in \mathbb{K}$ are arbitrary. So the quadratic forms $C^{i}$ that, a priori, are forms in the variables $x_{\tau+1}, \ldots, x_{N}$, actually depend only on the variables $x_{m+1}, \ldots, x_{N}$ and the result follows since $C_{0}, \ldots, C_{\tau}$ are linearly independent being the partial derivatives of $f$ with respect to the corresponding variable.

On the contrary by reversing the argument every cubic hypersurface with an equation of the form (21) is such that the polar quadric of a general point of $\mathbb{P}^{N}$ is tangent to $L$ along the common generator $M$. Moreover $\operatorname{codim}(Z)=1$ by Lemma 3.4 .

The next result is crucial to obtain a simplified canonical form for Special Perazzo Cubic Hypersurfaces. Our proof was completely inspired by the calculations made by Perazzo in [Pe, $\S 1.14-1.16$ p. 344-349], where as always we shall $\operatorname{suppose} \operatorname{codim}(Z)=1$, that is $p=0$ in Perazzo's notation.

Theorem 3.7. Let $X=V(f) \subset \mathbb{P}^{N}$ be an irreducible cubic hypersurface with vanishing hessian, not a cone, and such that $\operatorname{codim}(Z)=1$. Then the following conditions are equivalent:

i) $X \subset \mathbb{P}^{N}$ is a Special Perazzo Cubic Hypersurface with $\operatorname{dim}\left(Z^{*}\right)+1=\sigma$ and with

$$
L=\bigcap_{w \in W \text { general }} \mathbb{P}_{w}^{N-\operatorname{dim}\left(Z^{*}\right)}=\mathbb{P}^{N-\sigma}
$$

ii) $\operatorname{dim}\left(Z^{*}\right)=\sigma-1$ and the polar quadric $Q_{p}$ of a general point $p \in \mathbb{P}^{N}$ is tangent to the linear space $L=\mathbb{P}^{N-\sigma}$ along a common generator $M=\mathbb{P}^{\sigma}$ of $Q_{p}$ with

$$
\bigcap_{m \in M \backslash \operatorname{Vert}\left(Q_{p}\right)} T_{m} Q_{p}=L .
$$

Moreover $X \subset \mathbb{P}^{N}$ is projectively equivalent to

$$
V\left(\sum_{i=0}^{\sigma} x_{i} C^{i}\left(x_{N-\sigma+1}, \ldots, x_{N}\right)+D\left(x_{\sigma+1}, \ldots, x_{N}\right)\right) \subset \mathbb{P}^{N} .
$$

Proof. Let $X$ be a Special Perazzo Cubic Hypersurface and let

$$
<Z^{*}>=M=V\left(x_{\sigma+1}, \ldots, x_{N}\right) \subset L=V\left(x_{N-\sigma+1}, \ldots, x_{N}\right) .
$$

Since $M \subset \operatorname{Sing} X$ by Proposition 2.12 we deduce from Proposition 3.1 that

$$
f=\sum_{i=0}^{\sigma} x_{i} C^{i}\left(x_{\sigma+1}, \ldots, x_{N}\right)+D\left(x_{\sigma+1}, \ldots, x_{N}\right),
$$

with $C^{i}\left(x_{\sigma+1}, \ldots, x_{N}\right)$ a quadratic form for every $i=0, \ldots, \tau$ and with $D\left(x_{\tau+1}, \ldots, x_{N}\right)$ a cubic form.

In our hypothesis (12) yields $\mathbb{P}_{w}^{N-\operatorname{dim}\left(Z^{*}\right)}=\operatorname{Sing} Q_{w}$ for $w \in W=Z^{*}$ general. Since $Z^{*}$ is a hypersurface in $M$, for $m \in M$ general there exist $w_{1}, w_{2} \in Z^{*}$ general such that $m \in<$ $w_{1}, w_{2}>$. Then $L \subseteq \operatorname{Sing} Q_{m}$ because $L \subseteq \operatorname{Sing} Q_{w_{i}}$ for $i=1,2$. Therefore $L \subseteq \operatorname{Sing} Q_{m}$ for every $m \in M$.

By the previous expression of $f$ we get $V\left(C^{i}\right)=Q_{p_{i}} \subset \mathbb{P}^{N}$ with $p_{i}, i=0, \ldots, \sigma$, the $i$-th fundamental point of $M$. The condition $L \subseteq \operatorname{Sing} V\left(C^{i}\right)$ is equivalent to the fact that the quadratic forms $C^{i}$,s do not depend on the variables $x_{\sigma+1}, \ldots, x_{N-\sigma}$ for every $i=0, \ldots, \sigma$. Hence $f$ has an equation of the form (21) and from the last part of Theorem 3.6 we deduce that 
the general quadric $Q_{p}$ is tangent to $L$ along the generator $M$. From $L \subseteq \bigcap_{m \in M \backslash \operatorname{Vert}\left(Q_{p}\right)} T_{m} Q_{p}$ and from Lemma 3.4 we deduce that equality holds, proving ii).

Let us now assume ii) with $L=V\left(x_{N-\sigma+1}, \ldots, x_{N}\right)$ and $M=V\left(x_{\sigma+1}, \ldots, x_{N}\right)$. Let $p \in \mathbb{P}^{N}$ be a general point and let $q \in L_{p}=<p, L>$ be a general point. We claim that Sing $Q_{q}=\operatorname{Sing} Q_{p}$.

Indeed, if $p=[\mathbf{v}]$ and if $q=[\mathbf{u}]$, then $\mathbf{u}=\alpha \mathbf{v}+\beta \mathbf{z}$ with $[\mathbf{z}] \in L$ and $\alpha \neq 0$. Let $s=[\mathbf{s}]=\operatorname{Sing} Q_{q} \subset M \subset L$. From (21) we infer $L \subseteq \operatorname{Sing} Q_{t}$ for every $t \in L$, yielding

$$
\sum_{j=0}^{N} \frac{\partial^{2} f}{\partial x_{i} \partial x_{j}}(t) \mathbf{s}_{j}=0 \text { for every } i=0, \ldots, N .
$$

With the previous notation, we have for every $i=0, \ldots, N$ :

$$
\sum_{j=0}^{N} \frac{\partial^{2} f}{\partial x_{i} \partial x_{j}}(\mathbf{u}) \mathbf{s}_{j}=\alpha \sum_{j=0}^{N} \frac{\partial^{2} f}{\partial x_{i} \partial x_{j}}(\mathbf{v}) \mathbf{s}_{j}+\beta \sum_{j=0}^{N} \frac{\partial^{2} f}{\partial x_{i} \partial x_{j}}(\mathbf{z}) \mathbf{s}_{j}=\alpha \sum_{j=0}^{N} \frac{\partial^{2} f}{\partial x_{i} \partial x_{j}}(\mathbf{v}) \mathbf{s}_{j}
$$

where the last equality follows from (23). In conclusion $\operatorname{Sing} Q_{p}=\operatorname{Sing} Q_{q}$ for a general $q \in L_{p}$, as claimed.

Thus $\mathbb{P}^{N-\sigma+1}=L_{p} \subseteq \overline{\mathcal{P}_{X}^{-1}\left(\mathcal{P}_{X}(p)\right)}=\mathbb{P}^{N-\operatorname{dim}\left(Z^{*}\right)}$ so that equality holds since $\operatorname{dim}\left(Z^{*}\right)+1=$ $\sigma$ by hypothesis, concluding the proof of i).

The next result appears in $[\mathrm{Pe}, \S 1.17,1.18$ p. 348, 349, 350], with a small imprecision, because the determinantal variety found there is not necessarily irreducible, see also Example 2 below.

Theorem 3.8. Let notation be as above and let $X=V(f) \subset \mathbb{P}^{N}$ be a Special Perazzo Cubic Hypersurface with $f$ as in (22). Then $Z^{*}$ is an irreducible component of the determinantal hypersurface $\left.V\left(\operatorname{det}(A), x_{\sigma+1}, \ldots, x_{N}\right)\right) \subset M=\mathbb{P}^{\operatorname{dim}\left(Z^{*}\right)+1}$ with $A$ a $\sigma \times \sigma$ matrix of linear forms in the variables $x_{0}, \ldots, x_{\sigma}$. In particular $\operatorname{deg}\left(Z^{*}\right) \leq \sigma=\operatorname{dim}\left(Z^{*}\right)+1$

Proof. Let notation be as above, let $f$ be as in (22) and let $p=\left(y_{0}: \cdots: y_{N}\right)=[\mathbf{y}] \in \mathbb{P}^{N}$ be a general point. The point $\operatorname{Sing} Q_{p} \subset M$ has equations

$$
\sum_{j=0}^{N} \frac{\partial^{2} f}{\partial x_{i} \partial x_{j}}(\mathbf{y}) x_{j}=0
$$

$i=0, \ldots, N$, and

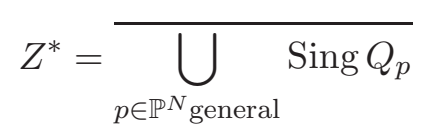

are the (implicit) parametric equations of $Z^{*}$ depending on $\mathbf{y}$. Since $Z^{*} \subset M=V\left(x_{\sigma+1}, \ldots, x_{N}\right) \subset$ $\mathbb{P}^{N}$, taking into account (22), these equations reduce to

$$
\sum_{j=0}^{\sigma} \frac{\partial C^{j}}{\partial x_{i}}\left(y_{N-\sigma+1}, \ldots, y_{N}\right) x_{j}=0=x_{\sigma+1}=\ldots=x_{N}
$$


$i=N-\sigma+1, \ldots, N$. Moreover by hypothesis the $\sigma \times(\sigma+1)$ matrix

$$
C\left(y_{N-\sigma+1}, \ldots, y_{N}\right)=\left[\frac{\partial C^{j}}{\partial x_{i}}\left(y_{N-\sigma+1}, \ldots, y_{N}\right)\right]
$$

has rank $\sigma$ for $\left(y_{N-\sigma+1}, \ldots, y_{N}\right)$ general because $\operatorname{Sing} Q_{p}$ is a point for $p \in \mathbb{P}^{N}$ general.

Let $C_{k, l}^{j}=\frac{\partial^{2} C^{j}}{\partial x_{k} \partial x_{l}}$. Then

$$
\frac{\partial C^{j}}{\partial x_{i}}\left(y_{N-\sigma+1}, \ldots, y_{N}\right)=\sum_{k=N-\sigma+1}^{N} C_{k, i}^{j} y_{k}
$$

and (24) can be written in the form

$$
\sum_{j=0}^{\sigma}\left(\sum_{k=N-\sigma+1}^{N} C_{k, i}^{j} y_{k}\right) x_{j}=0=x_{\sigma+1}=\ldots=x_{N},
$$

that is

$$
\sum_{k=N-\sigma+1}^{N}\left(\sum_{j=0}^{\sigma} C_{k, i}^{j} x_{j}\right) y_{k}=0=x_{\sigma+1}=\ldots=x_{N} .
$$

Let

$$
A=A\left(x_{0}, \ldots, x_{\sigma}\right)=\left[\sum_{j=0}^{\sigma} C_{k, i}^{j} x_{j}\right]
$$

with $k, i \in\{N-\sigma+1, \ldots, N\}$. Then $A$ is a $\sigma \times \sigma$ matrix of linear forms in the variables $x_{0}, \ldots, x_{\sigma}$ and $Z^{*} \subseteq V\left(\operatorname{det}(A), x_{\sigma+1}, \ldots, x_{N}\right) \subset M$. Indeed, for $r \in Z^{*}$ general, every $[\mathbf{y}] \in \operatorname{Sing} Q_{r}$ is a solution of (25). Thus (25) has non trivial solutions for $r \in Z^{*}$ general, yielding $\operatorname{det}(A(r))=0$ and $\left.Z^{*} \subseteq V\left(\operatorname{det}(A), x_{\sigma+1}, \ldots, x_{N}\right)\right)$.

The next example is a Special Perazzo Cubic Hypersurface $X \subset \mathbb{P}^{6}$ with $\operatorname{codim}(Z)=1$, $\operatorname{dim}\left(Z^{*}\right)=2$ and such that $Z^{*} \subset \mathbb{P}^{3}$ is a quadric hypersurface which is an irreducible component of the cubic surface $\left.V\left(\operatorname{det}(A), x_{\sigma+1}, \ldots, x_{N}\right)\right)$. Thus the inclusion

$$
\left.Z^{*} \subseteq V\left(\operatorname{det}(A), x_{\sigma+1}, \ldots, x_{N}\right)\right)
$$

proved in Theorem 3.8 can be strict in contrast to the claim made by Perazzo in [Pe, pg. 350] according to which $Z^{*}$ should be always of degree $\sigma$ for Special Perazzo Cubic Hypersurfaces.

Example 2. Let $X=V(f) \subset \mathbb{P}^{6}$ be the cubic hypersurface given by:

$$
f=x_{0} x_{4} x_{5}+x_{1} x_{4}^{2}+x_{2} x_{4} x_{6}+x_{3} x_{5} x_{6}
$$

We have $f_{0}=x_{4} x_{5}, f_{1}=x_{4}^{2}, f_{2}=x_{4} x_{6}$ e $f_{3}=x_{5} x_{6}$ so that

$$
f_{0} f_{2}=f_{1} f_{3} \text {. }
$$

Here we use the same notation as in Theorem 3.8. Thus $M=\mathbb{P}^{3}=V\left(x_{4}, x_{5}, x_{6}\right)$, For $i=4,5,6$ consider the system:

$$
\sum_{j=0}^{3} \frac{\partial^{2} f}{\partial x_{i} \partial x_{j}}(p) x_{j}=0=x_{4}=x_{5}=x_{6},
$$


with $p=\left(y_{0}: \ldots: y_{6}\right)$.

We get

$$
\left\{\begin{array}{c}
2 x_{1} y_{4}+x_{0} y_{5}+x_{2} y_{6}=0 \\
x_{0} p_{4}+0 y_{5}+x_{3} y_{6}=0 \\
x_{2} y_{4}+x_{3} y_{5}+0 y_{6}=0
\end{array}\right.
$$

Then

$$
\mathrm{A}=\left[\begin{array}{ccc}
2 x_{1} & x_{0} & x_{2} \\
x_{0} & 0 & x_{3} \\
x_{2} & x_{3} & 0
\end{array}\right]
$$

and $\operatorname{det}(A)=2 x_{0} x_{2} x_{3}-2 x_{1} x_{3}^{2}=2 x_{3}\left(x_{0} x_{2}-x_{1} x_{3}\right)$. Since $Z^{*}$ is irreducible and non linear we conclude that

$$
Z^{*}=V\left(x_{0} x_{2}-x_{1} x_{3}, x_{4}, x_{5}, x_{6}\right) \subset M \subset \mathbb{P}^{6} .
$$

\section{Cubics with Vanishing Hessian in $\mathbb{P}^{N}$ with $N \leq 6$}

The geometric structure and the canonical forms of cubic hypersurfaces with vanishing hessian and with $\operatorname{dim}\left(Z^{*}\right)=1$ is completely described in the next result.

Theorem 4.1. Let $X \subset \mathbb{P}^{N}$ be a cubic hypersurface with vanishing hessian, not a cone, with $\operatorname{dim}\left(Z^{*}\right)=1$. Then $N \geq 4, X$ is a Special Perazzo Cubic Hypersurface and we have:

1) $\operatorname{codim}(Z)=1$;

2) $S Z^{*}=\mathbb{P}^{2} \subseteq \operatorname{Sing} X$

3) $Z^{*}$ is a conic;

4) If $N=4$, then $X$ is projectively equivalent to $S(1,2)^{*}$;

5) $X$ is projectively equivalent to $V\left(x_{0} x_{N-1}^{2}+2 x_{1} x_{N-1} x_{N}+x_{2} x_{N}^{2}+D\left(x_{3}, x_{4}, \ldots, x_{N}\right)\right)$.

Proof. We can suppose $N \geq 4$ since cubic surfaces with vanishing hessian are easily seen to be cones. The dual of a projective curve, not a line, is a hypersurface so that by Reflexivity we get $\operatorname{codim}(Z)=1$. Thus $X$ is a Special Perazzo Cubic Hypersurface since the fibers of the Perazzo map are hyperplanes and hence $<Z^{*}>\subseteq$ Sing $X$.

Since a pencil of quadrics generated by two quadrics of rank one contains only two quadrics of rank one we deduce $\operatorname{deg}\left(Z^{*}\right)=2$ (this can be also deduced from Theorem 3.8) and $\left\langle Z^{*}\right\rangle=$ $\mathbb{P}^{2}$.

If $N=4$, then $X$ is a scroll in $\mathbb{P}^{2}$ tangent to $Z^{*}$ and $X^{*}$ is a surface scroll with line directrix $\left.<Z^{*}\right\rangle^{*}$ over the dual conic of $Z^{*}$ in its linear span. Therefore $X^{*}$ is projectively equivalent to the cubic rational normal scroll $S(1,2) \subset \mathbb{P}^{4}$, proving 4 ).

Part 5) is a particular case of (22) taking into account that $C_{0}, C_{1}, C_{2}$ is a basis of $\mathbb{K}\left[x_{0}, x_{1}, x_{2}\right]_{2}$ because $f_{i}=C_{i}, i=0, \ldots, 2$ are linearly independent. Therefore modulo a change of coordinates in $\mathbb{P}^{N}$ acting on $V\left(x_{N-1}, x_{N}\right)$ as the identity map, we can take $C_{0}=x_{N-1}^{2}, C_{1}=2 x_{N-1} x_{N}$ and $C_{2}=x_{N}^{2}$. Then $Z=V\left(2 z_{0} z_{2}-z_{1}^{2}\right) \subset \mathbb{P}^{N *}$ and $Z^{*}=V\left(2 x_{0} x_{2}-x_{1}^{2}, x_{3}, \ldots, x_{N}\right) \subset \mathbb{P}^{N}$.

As recalled above for $N \leq 3$ a cubic hypersurface with vanishing hessian is easily seen to be a cone. We shall now consider the cases $N=4,5,6$. 
4.1. Cubics with Vanishing Hessian in $\mathbb{P}^{4}$. The next Theorem is essentially a compilation of classical results, more or less known nowadays, see [Pe], CRS] and [GR].

Theorem 4.2. Let $X \subset \mathbb{P}^{4}$ be an irreducible cubic hypersurface with vanishing hessian, not a cone. Then

i) $(\operatorname{Sing} X)_{\text {red }}=\mathbb{P}^{2}$;

ii) $X^{*} \simeq S(1,2) \subset \mathbb{P}^{4}$;

iii) $X$ is projectively equivalent to a linear external projection of $\operatorname{Seg}(1,2) \subset \mathbb{P}^{5}$;

iv) $X$ is projectively equivalent to $V\left(x_{0} x_{3}^{2}+2 x_{1} x_{3} x_{4}+x_{2} x_{4}^{2}\right) \subset \mathbb{P}^{4}$.

Proof. First of all let us remark that $\operatorname{codim}(Z)=1$. On the contrary by cutting $X$ with a general hyperplane $H$ and by projecting $Z$ from the general point $[H]$ we would obtain a cubic hypersurface in $\mathbb{P}^{3}$ with vanishing hessian, which would be a cone. This would imply that $X$ is a cone. Thus $\operatorname{codim}(Z)=1$ and from (15) we get $\operatorname{dim}\left(Z^{*}\right)=1$. All conclusions now follow from Theorem 4.1, see also Example 1, except for iv). Theorem 4.1 implies that $X$ is projectively equivalent to $Y=V\left(x_{0} x_{3}^{2}+2 x_{1} x_{3} x_{4}+x_{2} x_{4}^{2}+D\left(x_{3}, x_{4}\right)\right) \subset \mathbb{P}^{4}$ with $D\left(x_{3}, x_{4}\right)=\left(a x_{3}+b x_{4}\right) x_{3}^{2}+\left(c x_{3}+d x_{4}\right) x_{4}^{2}$. The projective transformation $x_{0}^{\prime}=x_{0}+a x_{3}+b x_{4}$, $x_{1}^{\prime}=x_{1}, x_{2}^{\prime}=x_{2}+c x_{3}+d x_{4}, x_{3}^{\prime}=x_{3}$ and $x_{4}^{\prime}=x_{4}$ sends $Y$ into $V\left(x_{0} x_{3}^{2}+2 x_{1} x_{3} x_{4}+x_{2} x_{4}^{2}\right)$, as claimed.

\subsection{Cubics with vanishing hessian in $\mathbb{P}^{5}$.}

Theorem 4.3. Let $X=V(f) \subset \mathbb{P}^{5}$ be a cubic hypersurface with vanishing hessian, not a cone. Then:

i) $X$ is a Special Perazzo Cubic Hypersurface such that $Z^{*}$ is a conic so that $X$ is a scroll in $\mathbb{P}^{2}$ tangent to the conic $Z^{*}$;

ii) $X$ is projectively equivalent to $V\left(x_{0} x_{4}^{2}+2 x_{1} x_{4} x_{5}+x_{2} x_{5}^{2}+D\left(x_{3}, x_{4}, x_{5}\right)\right) \subset \mathbb{P}^{5}$ with $D\left(x_{3}, x_{4}, x_{5}\right)$ a cubic form.

Proof. Since the polar image of every cubic hypersurface with vanishing hessian in $\mathbb{P}^{4}$ is a quadric hypersurface, reasoning as in the proof of Theorem 4.2, we deduce $\operatorname{codim}(Z)=1$. Therefore $\operatorname{dim}\left(Z^{*}\right) \leq 2$ by (15). If $\operatorname{dim}\left(Z^{*}\right)=2$, then either $X$ is a Special Perazzo Cubic Hypersurface or two general fibers of the Perazzo map span $\mathbb{P}^{5}$. In both cases we would deduce $\left\langle Z^{*}>\subset \operatorname{Sing}(X)\right.$ and $X$ would be a cone by Proposition 3.1 Thus $\operatorname{dim}\left(Z^{*}\right)=1$ and we can apply Theorem 4.1 to conclude.

4.3. Cubics with vanishing hessian in $\mathbb{P}^{6}$. Let us remark that since the polar image of every cubic hypersurface in $\mathbb{P}^{5}$ with vanishing hessian, not a cone, is a quadric hypersurface we deduce that $\operatorname{codim}(Z)=1$ for each cubic hypersurface in $\mathbb{P}^{6}$ with vanishing hessian. Therefore (15) yields $\operatorname{dim}\left(Z^{*}\right) \leq 2$. 
4.3.1. The case $\operatorname{dim}\left(Z^{*}\right)=1$.

Theorem 4.4. Let $X \subset \mathbb{P}^{6}$ be an irreducible cubic hypersurface with vanishing hessian, not a cone, and such that $\operatorname{dim}\left(Z^{*}\right)=1$. Then $X$ is a Special Perazzo Cubic Hypersurface projectively equivalent to $V\left(x_{0} x_{5}^{2}+2 x_{1} x_{5} x_{6}+x_{2} x_{6}^{2}+D\left(x_{3}, x_{4}, x_{5}, x_{6}\right)\right.$, where $D$ is a cubic form in the variables $x_{3}, x_{4}, x_{5}$, and $x_{6}$.

Furthermore the surface $V\left(D, x_{0}, x_{1}, x_{2}\right) \subset V\left(x_{0}, x_{1}, x_{2}\right)$ is not singular along the line $T=V\left(x_{0}, x_{1}, x_{2}, x_{5}, x_{6}\right)$.

Proof. All the conclusions follow from Theorem 4.1 except the condition of non singularity of $V(D)$ along $T$ which is necessary for $X$ not to be a cone as one verifies by computing the derivatives of the general canonical form.

Remark 4.5. Using the canonical form in Theorem 4.1 a direct computation shows that there are only three possibilities for $(\operatorname{Sing} X)_{\text {red }}$ in Theorem 4.4,

If $T \cap \operatorname{Sing} V(D)=\emptyset$, then $(\operatorname{Sing} X)_{\text {red }}=\mathbb{P}^{2}$. If $T \cap \operatorname{Sing} V(D) \neq \emptyset$, then either the intersection consists of two points and $(\operatorname{Sing} X)_{\text {red }}=\mathbb{P}_{1}^{3} \cup \mathbb{P}_{2}^{3}$; or it consists of a point and $(\operatorname{Sing} X)_{\mathrm{red}}=\mathbb{P}^{3}$.

By analyzing only the canonical form Perazzo did not distinguish the three previous cases. The next result describes the different geometry of $X$ occurring in the three distinct cases.

Theorem 4.6. Let $X \subset \mathbb{P}^{6}$ be a cubic hypersurface having vanishing hessian, not a cone, with $\operatorname{dim}\left(Z^{*}\right)=1$. Then $X$ is a Special Perazzo Cubic Hypersurface which is a scroll in $\mathbb{P}^{2}$ tangent to the conic $Z^{*}$.

Moreover if $\left\langle Z^{*}>\subsetneq(\text { Sing } X)_{\text {red }}\right.$ then one of the following conditions holds:

i) If $(\operatorname{Sing} X)_{\text {red }}=\mathbb{P}^{3}$, then $X$ is also a scroll in $\mathbb{P}^{3}$ tangent to the conic $Z^{*}$.

ii) If $(\operatorname{Sing} X)_{\text {red }}=\mathbb{P}_{1}^{3} \cup \mathbb{P}_{2}^{3}$, then there exist two different structures of scroll in $\mathbb{P}^{3}$ tangent to $Z^{*}$ such that the members of the two structures passing through a general point $x \in X$ intersect in a $\mathbb{P}_{x}^{2}$ which is tangent to $Z^{*}$, yielding the original structure of scroll in $\mathbb{P}^{2}$ tangent to $Z^{*}$.

Proof. By Theorem 4.1 we know that $X$ has a structure of scroll in $\mathbb{P}^{2}$ tangent to the conic $Z^{*}$.

By Remark 4.5 if $<Z^{*}>\subsetneq(\operatorname{Sing} X)_{\text {red }}$, then either $(\operatorname{Sing} X)_{\text {red }}=\mathbb{P}^{3}$ or $(\operatorname{Sing} X)_{\text {red }}=$ $\mathbb{P}_{1}^{3} \cup \mathbb{P}_{2}^{3}$. In both cases the $\mathbb{P}^{4}$ 's passing through a $\mathbb{P}^{3}$ contained in Sing $X$ induce on $X$ a structure of scroll in $\mathbb{P}^{3}$ tangent to $Z^{*}$. Moreover in case ii) the $\mathbb{P}^{3}$ 's of each scroll structure passing through a general $x \in X$ are tangent to $Z^{*}$ at the same point $\mathcal{P}_{X}(x)$ so that their intersection $\mathbb{P}_{x}^{2}$ is also tangent to $Z^{*}$ at $\mathcal{P}_{X}(x)$. By construction this structure coincides with the one described at the beginning of the proof.

4.3.2. The case $\operatorname{dim}\left(Z^{*}\right)=2$.

Theorem 4.7. Let $X \subset \mathbb{P}^{6}$ be a cubic hypersurface with vanishing hessian, not a cone, with $\operatorname{dim}\left(Z^{*}\right)=2$. Then

i) $X$ is a Special Perazzo Cubic Hypersurface which is a scroll in $\mathbb{P}^{3}$ tangent to the surface $Z^{*}$, which is either a quadric or a cubic surface in $\mathbb{P}^{3}$; 
ii) $X$ is projectively equivalent to

$$
V\left(\sum_{i=0}^{3} x_{i} C^{i}\left(x_{4}, x_{5}, x_{6}\right)+D\left(x_{4}, x_{5}, x_{6}\right)\right),
$$

where $C^{0}, C^{1}, C^{2}$, and $C^{3}$ are quadratic forms and $D$ is a cubic form;

iii) $X$ is the dual variety of a scroll in $\mathbb{P}^{2}$ over a surface $\widetilde{Z} \subset \mathbb{P}^{3}$ which is projectively equivalent to the dual of $Z^{*}$ in its linear span. Moreover $X^{*}$ cuts a general generator of $Z$, which is a cone over $\widetilde{Z}$ with vertex $<Z^{*}>^{*}=\mathbb{P}^{2}$, along a plane of the ruling.

Proof. Let $\mathbb{P}_{r_{i}}^{4}=\overline{\mathcal{P}_{X}^{-1}\left(r_{i}\right)}, i=1,2$, be two general fibers of the Perazzo map of $X \subset \mathbb{P}^{6}$. There are two possibilities: either $<\mathbb{P}_{r_{1}}^{4}, \mathbb{P}_{r_{2}}^{4}>=\mathbb{P}^{5}$ (or equivalently $\mathbb{P}_{r_{1}}^{4} \cap \mathbb{P}_{r_{2}}^{4}=\mathbb{P}^{3}$ ) or $<\mathbb{P}_{r_{1}}^{4}, \mathbb{P}_{r_{2}}^{4}>=\mathbb{P}^{6}$ (or equivalently $\mathbb{P}_{r_{1}}^{4} \cap \mathbb{P}_{r_{2}}^{4}=\mathbb{P}^{2}$ ).

In the first case $X$ is a Special Perazzo Cubic Hypersurface by Lemma 2.10. In the second case Proposition 2.13 implies $\left\langle Z^{*}\right\rangle=\mathbb{P}^{3} \subseteq \operatorname{Sing} X$ but this case cannot exist by Proposition 2.12. Theorem 3.8 yields $\operatorname{deg}\left(Z^{*}\right) \leq 3$, concluding the proof of i) while part ii) follows from Theorem 3.7

\section{EXAmples in HigheR Dimensions}

The classification of cubic hypersurfaces with vanishing hessian $X \subset \mathbb{P}^{N}$ for $N \leq 6$ showed that they all have $\operatorname{codim}(Z)=1$ and that they are Special Perazzo Cubic Hypersurfaces.

In [CRS, §2] two series of examples of hypersurfaces with vanishing hessian $X \subset \mathbb{P}^{N}$, $N \geq 5$, of sufficiently high degree and such that $\operatorname{codim}(Z)>1$ have been constructed, see [CRS, Proposition 2.15, Remark 2.16, Remark 2.19].

We now construct some examples of cubic hypersurfaces in $\mathbb{P}^{N}, N \geq 7$, with $\operatorname{codim}(Z)>1$. Later on we shall provide also examples of cubic hypersurfaces with vanishing hessian not a cone in $\mathbb{P}^{N}$ with $N=7,13,25$ which are not Special Perazzo Cubic Hypersurfaces.

First we give two different constructions of cubic hypersurfaces with vanishing hessian, not cones, from examples in lower dimension dubbed concatenation and juxtaposition. By repeated use of these methods one can prove that for a fixed $\alpha \in \mathbb{N}$ there exists $N_{0}=N_{0}(\alpha)$ such that for every $N \geq N_{0}$ there exists a cubic hypersurface $X \subset \mathbb{P}^{N}$ with vanishing hessian, not a cone, and such that $\operatorname{codim}(Z)=\alpha$.

Example 3. Given $g=x_{0} x_{3}^{2}+x_{1} x_{3} x_{4}+x_{2} x_{4}^{2}$ let $\tilde{g}=x_{2} x_{4}^{2}+x_{5} x_{4} x_{7}+x_{6} x_{7}^{2}$. We define the concatenation of $g$ and $\tilde{g}$ as the form $f$ obtained by summing up the monomials without repetition, that is

$$
f=x_{0} x_{3}^{2}+x_{1} x_{3} x_{4}+x_{2} x_{4}^{2}+x_{5} x_{4} x_{7}+x_{6} x_{7}^{2} .
$$

Then $V(f) \subset \mathbb{P}^{7}$ is a cubic hypersurface with vanishing hessian, not a cone. Indeed, letting $f_{i}=\frac{\partial f}{\partial x_{i}}$ one easily verifies that the $f_{i}$ 's are linearly independent so that $X$ is not a cone and $Z$ is non degenerate. Moreover

$$
f_{0} f_{2}=f_{1}^{2} \quad \text { and } \quad f_{2} f_{6}=f_{5}^{2}
$$

yielding $\operatorname{codim}(Z) \geq 2$ and $\operatorname{deg}(Z) \geq 3$. Thus $\operatorname{codim}(Z)=2$ since the polar image of every cubic hypersurface with vanishing hessian in $\mathbb{P}^{5}$, not a cone, is a quadric hypersurface. 
Example 4. Let $g=x_{0} x_{3}^{2}+x_{1} x_{3} x_{4}+x_{2} x_{4}^{2}$ and let

$$
f=x_{0} x_{3}^{2}+x_{1} x_{3} x_{4}+x_{2} x_{4}^{2}+x_{5} x_{8}^{2}+x_{6} x_{7} x_{8}+x_{7} x_{9}^{2}
$$

be the cubic form obtained by the juxtaposition of $g$ with itself, that is $f$ is the sum of $g$ and $\tilde{g}=x_{5} x_{8}^{2}+x_{6} x_{7} x_{8}+x_{7} x_{9}^{2}$ obtained by shifting the indexes of $g$.

As above it is possible to verify immediately that $X=V(f) \subset \mathbb{P}^{9}$ is not a cone and that the following algebraic relations hold $f_{0} f_{2}=f_{1}^{2}, f_{5} f_{7}=f_{6}^{2}$.

Example 5. Let $g=x_{0} x_{3}^{2}+x_{1} x_{3} x_{4}+x_{2} x_{4}^{2}$ and let

$$
f=x_{0} x_{3}^{2}+x_{1} x_{3} x_{4}+x_{2} x_{4}^{2}+x_{5} x_{8}^{2}+x_{6} x_{7} x_{8}+x_{7} x_{9}^{2}+x_{1-} x_{13}^{2}+x_{11} x_{13} x_{14}+x_{12} x_{14}^{2}
$$

be the cubic form obtained by the juxtaposition of $g$ with itself two times.

Then $X=V(f) \subset \mathbb{P}^{12}$ is not a cone and the following algebraic relations hold: $f_{0} f_{2}=f_{1}^{2}$, $f_{5} f_{7}=f_{6}^{2}, f_{10} f_{12}=f_{11}^{2}$.

We conclude with an example of a cubic hypersurface $X \subset \mathbb{P}^{7}$ with vanishing hessian, not a cone, which is not a Special Perazzo Cubic Hypersurface.

Example 6. Let

$$
g=\operatorname{det}\left(\begin{array}{lll}
x_{0} & x_{1} & x_{2} \\
x_{3} & x_{4} & x_{5} \\
x_{6} & x_{7} & x_{8}
\end{array}\right) .
$$

Then $R=V(g) \subset \mathbb{P}^{8}$ is a cubic hypersurface. If we identify $\mathbb{P}^{8}$ with $\mathbb{P}\left(\mathbb{M}_{3 \times 3}(\mathbb{K})\right)$, then $R$ is the locus of matrices of rank at most two and it is naturally identified with the secant variety of $W=\mathbb{P}^{2} \times \mathbb{P}^{2} \subset \mathbb{P}^{8}$, the locus of matrices of rank 1. Moreover Sing $R=W$ as schemes.

The polar map $\Phi_{R}: \mathbb{P}^{8} \rightarrow \mathbb{P}^{8}$ is a birational involution sending a matrix $p=[A]$ to its cofactor matrix. Since the cofactor matrix of a rank two matrix has rank one and since $\Phi_{R \mid R}=\mathcal{G}_{R}$, we deduce that $\overline{\Phi_{R}(R)}=\overline{\mathcal{G}_{R}(R)}=R^{*}$ is naturally identified with $W$. By the previous description $\Phi_{R}$ is an isomorphism on $\mathbb{P}^{8} \backslash R$ and the closure of every positive dimensional fiber of $\Phi_{R}$ (and hence of every fiber of $\mathcal{G}_{R}$ ) is a $\mathbb{P}^{3}$. Indeed by homogeneity it is sufficient to verify this for $\overline{\Phi_{R}^{-1}(q)}$ with $q=(0: 0: 0: 0: 0: 0: 0: 0: 1)$. The $3 \times 3$ matrices mapped by $\Phi_{R}$ to $q$ are exactly the rank two matrices $X=\left[x_{i, j}\right]$ having $x_{2}=x_{3}=x_{5}=x_{6}=x_{7}=x_{8}=0$, i.e. $\overline{\Phi_{R}^{-1}(q)}=V\left(x_{2}, x_{5}, x_{6}, x_{7}, x_{8}\right)$ is the closure of the orbit of

$$
p=\left(\begin{array}{lll}
1 & 0 & 0 \\
0 & 1 & 0 \\
0 & 0 & 0
\end{array}\right)
$$

under the natural action.

Let

$$
f=\operatorname{det}\left(\begin{array}{ccc}
x_{0} & x_{1} & x_{2} \\
x_{3} & x_{4} & x_{5} \\
x_{6} & x_{7} & 0
\end{array}\right) .
$$

Then $x_{8}=0$ is the equation of $T_{p} R$ with $p \in R$ the point defined in (27). 
Let

$$
X=R \cap T_{p} R=V(f) \subset \mathbb{P}^{7}=V\left(x_{8}\right) \subset \mathbb{P}^{8} .
$$

Then $X \subset \mathbb{P}^{7}$ is a cubic hypersurface with vanishing hessian, not a cone. Indeed the partial derivatives $f_{i}$ 's are linearly independent and we have

$$
f_{0} f_{4}=x_{2} x_{5} x_{6} x_{7}=f_{1} f_{3} .
$$

More precisely $Z=\overline{\Phi_{X}\left(\mathbb{P}^{7}\right)}=V\left(y_{0} y_{4}-y_{1} y_{3}\right) \subset\left(\mathbb{P}^{7}\right)^{*}$, is a rank four quadric with vertex $V=V\left(y_{0}, y_{1}, y_{3}, y_{4}\right)$. Thus

$$
Z^{*}=V\left(x_{0} x_{4}-x_{1} x_{3}, x_{2}, x_{5}, x_{6}, x_{7}\right) \subset<Z^{*}>=V\left(x_{2}, x_{5}, x_{6}, x_{7}\right)=\mathbb{P}^{3} .
$$

Hence $\left\langle Z^{*}>=\overline{\Phi_{R}^{-1}(q)}\right.$ is the fiber of the Gauss map of $R$ passing through $p$, that is the contact locus on $R$ of the hyperplane $T_{p} R$, yielding $<Z^{*}>\subseteq \operatorname{Sing}(X)$ (a fact which can be verified also directly). The variety $Z^{*}$ is thus the locus of secant and tangent lines to $W$ passing through $p \in R=S W$.

The hypersurface $X$ is singular also along $\operatorname{Sing} R \cap T_{p} R=W \cap T_{p} R$. We claim that $(\operatorname{Sing} X)_{\text {red }}=<Z^{*}>\cup Y_{1} \cup Y_{2}$, where each $Y_{i} \subset<Y_{i}>=\mathbb{P}^{5}$ is a Segre 3 -fold $\mathbb{P}^{1} \times \mathbb{P}^{2}$ and where $\left\langle Y_{1}>\cap<Y_{2}\right\rangle=\left\langle Z^{*}>\right.$. Indeed $T_{p} R \cap W$ is a hyperplane section of $W$ so that it has degree 6. Moreover $T_{p} R \cap W$ contains the following two Segre 3-folds:

$$
\operatorname{rk}\left(\begin{array}{ccc}
x_{0} & x_{1} & x_{2} \\
x_{3} & x_{4} & x_{5} \\
0 & 0 & 0
\end{array}\right)=1
$$

lying in $V\left(x_{6}, x_{7}\right)=\mathbb{P}^{5} \subset \mathbb{P}^{7}$ and

$$
\operatorname{rk}\left(\begin{array}{lll}
x_{0} & x_{1} & 0 \\
x_{3} & x_{4} & 0 \\
x_{6} & x_{7} & 0
\end{array}\right)=1
$$

lying in $V\left(x_{2}, x_{5}\right)=\mathbb{P}^{5} \subset \mathbb{P}^{7}$.

Let $\mathcal{P}_{X}: \mathbb{P}^{7} \rightarrow Z^{*}$ be the Perazzo map of $X$. Thus for $w \in W=Z^{*}$ general we have $\overline{\mathcal{P}_{X}^{-1}(w)}=\mathbb{P}_{w}^{5}$. More precisely if $w \in Z^{*}, w=\left(a_{0}: a_{1}: 0: a_{3}: a_{4}: 0: 0: 0\right)$ with $a_{0} a_{4}-a_{1} a_{3}=0$, then:

$$
Q_{w}=\left(\begin{array}{cccccccc}
0 & 0 & 0 & 0 & 0 & 0 & 0 & 0 \\
0 & 0 & 0 & 0 & 0 & 0 & 0 & 0 \\
0 & 0 & 0 & 0 & 0 & 0 & -a_{4} & a_{3} \\
0 & 0 & 0 & 0 & 0 & 0 & 0 & 0 \\
0 & 0 & 0 & 0 & 0 & 0 & 0 & 0 \\
0 & 0 & 0 & 0 & 0 & 0 & a_{1} & -a_{0} \\
0 & 0 & -a_{4} & 0 & 0 & a_{1} & 0 & 0 \\
0 & 0 & a_{3} & 0 & 0 & -a_{0} & 0 & 0
\end{array}\right)
$$

This matrix has rank two due to the relation $a_{0} a_{4}-a_{1} a_{3}=0$ and $\operatorname{Sing} Q_{w}=V\left(-a_{4} x_{6}+\right.$ $\left.a_{3} x_{7},-a_{4} x_{2}+a_{1} x_{5}\right)$ for $w$ general. Therefore two general fibers of the Perazzo map intersect in a $\mathbb{P}^{3}$ and $X=V(f) \subset \mathbb{P}^{7}$ is not a Special Perazzo Cubic Hypersurface.

Let us remark that $X^{*} \subset Z \subset\left(\mathbb{P}^{7}\right)^{*}$ is a 4-fold which by duality is the projection of $R^{*}$ from the point $\mathcal{G}_{R}(p)=q=(0: 0: \ldots: 0: 1)$. One can alternatively deduce $\operatorname{dim}\left(X^{*}\right)=4$ by 
first observing that $T_{p} R$ cuts a general fiber of the Gauss map of $R$ in a $\mathbb{P}^{2}$, which becomes the general fiber of the Gauss map of $X$ so that $\operatorname{dim}\left(X^{*}\right)=\operatorname{dim}(X)-2=4$.

To see the vanishing of the hessian of $X$ geometrically one remarks that $\Phi_{R}\left(T_{p} R\right)=Q \subset \mathbb{P}^{8}$ is a quadric singular at $\Phi_{R}(p)$. Indeed, the restriction of $\Phi_{R}$ to $T_{p} R$ is birational onto the image, which is a quadric hypersurface since $\Phi_{R}$ is given by quadratic equations and it is an involution; moreover the general positive dimensional fiber of the restriction of $\Phi_{R}$ to $T_{p} R$ is two dimensional while the fiber of the Gauss map through $p$ has dimension three. Thus projecting $Q$ from $\Phi_{R}(p)$ one obtains a quadric hypersurface $Q \subset \mathbb{P}^{7}$ containing $\Phi_{X}\left(\mathbb{P}^{7}\right)$. Thus $X \subset \mathbb{P}^{7}$ has vanishing hessian and it is not difficult to prove that $Z_{X}=Q$, also by direct computations as seen above.

Remark 5.1. One can construct in a similar way examples of cubic hypersurfaces with vanishing hessian, not cones, such that $\operatorname{codim}(Z)=1$ and $\operatorname{codim}\left(X^{*}, Z\right)>1$. Indeed by taking as $R$ the secant variety to one of the two Severi varieties $W=\mathbb{G}(1,5) \subset \mathbb{P}^{14}$, respectively $W=E_{6} \subset \mathbb{P}^{26}$, and by considering their section by a tangent hyperplane one gets examples of cubic hypersurfaces $X \subset \mathbb{P}^{N}$ with $N=13$, respectively $N=25$, such that $Z_{X}$ is a quadric hypersurface while $X^{*}$ has dimension 8 , respectively 16 . Thus in the first case $\operatorname{codim}\left(X^{*}, Z\right)=4$ while in the second case $\operatorname{codim}\left(X^{*}, Z\right)=8$.

Let us recall that $\operatorname{dim}(Z)=\operatorname{rkHess}_{X}-1$ while it is not difficult to prove that $\operatorname{dim}\left(X^{*}\right)=$ $\operatorname{rk}_{f} \operatorname{Hess}_{X}-2$, where $\operatorname{rk}_{f} \operatorname{Hess}_{X}$ denotes the rank of the matrix Hess $X$ modulo the ideal $(f)$.

For the cubic hypersurfaces $X=V(f)=S W \subset \mathbb{P}^{\frac{3 n}{2}+1}, n=4,8,16$, deduced from the

corresponding Severi varieties $W^{n} \subset \mathbb{P}^{\frac{3 n}{2}+2}$, we have $\mathrm{rkHess}_{X}=\frac{3 n}{2}+1$ and $\mathrm{rk}_{f} \operatorname{Hess}_{X}=n+2$ since $\operatorname{codim}\left(X^{*}, Z\right)=\frac{n}{2}$. Thus for $n=4,8,16$ we have $\operatorname{rkHess}_{X}>\operatorname{rk}_{f} \operatorname{Hess}_{X}$, something which is somehow unexpected and which can occur only for non Special Cubic Perazzo Hypersurfaces.

In general it seems quite difficult to construct examples of (cubic) hypersurfaces with vanishing hessian, not cones, such that $\operatorname{codim}\left(X^{*}, Z\right)$ can be arbitrarily large. We plan to come back to this intriguing problem elsewhere.

\section{ACKNowledgements}

Our collaboration began several years ago in Recife while we were trying to understand, revisit and generalize the ideas contained in $\mathrm{Pe}$. The first author was supported by CAPES and FACEPE while the second author by CNPq, by the University of Catania and by PRIN "Geometria delle Varietà Algebriche". We would like to thank Ciro Ciliberto, Thiago Fassarella, Riccardo Re and Aron Simis for many interesting discussions on these topics along the years.

We are deeply indebted to the referee who read very carefully the whole paper providing many important remarks and suggestions which allowed us to improve significantly the exposition and to eliminate a lot of imprecisions, both mathematical and lexical. Due to his(/her) contributions our original rough manuscript turned into a mathematical paper.

\section{REFERENCES}

[AG1] M. A. Akivis, V. V. Goldberg, Differential Geometry of Varieties with Degenerate Gauss Maps, CMS books in Mathematics, 2004. 
[AG2] M. A. Akivis, V. V. Goldberg, Smooth Lines on Projective Planes over Two-Dimensional Algebras and Submanifolds with Degenerate Gauss Maps Beiträge zur Algebra und Geometrie "Contributions to Algebra and Geometry", Volume 44 (2003), No. 1, 165-178.

[AGL] M. A. Akivis, V. V. Goldberg, J. M. Landsberg On the structure of varieties with degenerate Gauss mappings, arXiv:math-9908079v2.

[Al] A. Alzati, Special linear systems and syzygies, Collect. Math. 59 (2008), 239-254.

[Ci] C. Ciliberto, Ipersuperficie algebriche a punti parabolici e relative hessiane, Rend. Acc. Naz. Scienze 98 (1979-80), 25-42.

[CRS] C. Ciliberto, F. Russo, A. Simis, Homaloidal hypersurfaces and hypersurfaces with vanishing Hessian, Advances in Mathematics, 218, (2008), 1759-1805.

[De] L. Degoli, Sui sistemi lineari di quadriche riducibili ed irriducibili a Jacobiana identicamente nulla, Collect. Math. 35 (1984), 131-148.

[Do] I. Dolgachev, Classical Algebraic Geometry - A modern view, Cambridge University Press, 2012.

[Fr1] A. Franchetta, Forme algebriche sviluppabili e relative hessiane, Atti Acc. Lincei 10 (1951), 1-4.

[Fr2] A. Franchetta, Sulle forme algebriche di $S_{4}$ aventi hessiana indeterminata, Rend. Mat. 13 (1954), $1-6$.

[FP] G. Fischer, J. Piontkowski, Ruled varieties- An introduction to algebraic differential geometry, Advanced Lectures in Mathematics. Friedr. Vieweg \& Sohn, Braunschweig, 2001. x+142 pp.

[FW] G. Fischer, H. Wu, Developable complex analytic submanifolds, Inter. Jour. Math. 6 (1995), $229-272$.

[GR] A. Garbagnati, F. Repetto, A geometrical approach to Gordan-Noether's and Franchetta's contributions to a question posed by Hesse, Collect. Math., 60 (2009), 27-41.

[GN] P. Gordan, M. Nöther, Ueber die algebraischen Formen, deren Hesse'sche Determinante identisch verschwindet, Math. Ann. 10 (1876), 547-568.

[He1] O. Hesse, Über die Bedingung, unter welche eine homogene ganze Function von n unabhángigen Variabeln durch Lineäre Substitutionen von $n$ andern unabhángigenVariabeln auf eine homogene Function sich zurück-führen lässt, die eine Variable weniger enthält, J. reine angew. Math. 42 (1851), 117-124.

[He2] O. Hesse, Zur Theorie der ganzen homogenen Functionen, J. reine angew. Math. 56 (1859), $263-269$.

[La] J.M. Landsberg, On degenerate secant and tangential varieties and local differential geometry, Duke Math. J. 85 (1996), 605-634.

[Lo] C. Lossen, When does the Hessian determinant vanish identically? (On Gordan and Noether's Proof of Hesse's Claim), Bull. Braz. Math. Soc. 35 (2004), 71-82.

[Pe] U. Perazzo, Sulle varietà cubiche la cui hessiana svanisce identicamente, Giornale di Matematiche (Battaglini) 38 (1900), 337-354.

[Pt1] R. Permutti, Su certe forme a hessiana indeterminata, Ricerche di Mat. 6 (1957), 3-10.

[Pt2] R. Permutti, Sul teorema di Hesse per forme sopra un campo a caratteristica arbitraria, Le Matematiche 13 (1963), 115-128.

[Pt3] R. Permutti, Su certe classi di forme a hessiana indeterminata, Ricerche di Mat. 13 (1964), 97-105.

[Se1] B. Segre, Bertini forms and hessian matrices, J. London Math. Soc. 26 (1951), 164-176.

[Se2] B. Segre, Some Properties of Differentiable Varieties and Transformations, Erg. Math., SpringerVerlag, 1957.

[Se3] B. Segre, Sull' hessiano di taluni polinomi (determinanti, pfaffiani, discriminanti, risultanti, hessiani) I, II, Atti Acc. Lincei 37 (1964), 109-117 and 215-221.

[Seg1] C. Segre, Su una classe di superficie degli iperspazi legate colle equazioni lineari alle derivate partziali di 2 ordine, Atti Accad. Sci. Torino Cl. Sci. Fis. Mat. Natur. 42 (1906-1907), 1047-1079.

[Seg2] C. Segre, Preliminari di una teoria delle varieta luoghi di spazi, Rend. Circ. Mat. Palermo 30 (1910), 87-121.

[Seg3] C. Segre, Ricerche sui fasci di coni quadrici in uno spazio lineare qualunque, Atti della R. Acc. delle Scienze di Torino, Vol. XIX. (1884). 
Universidade Federal Rural de Pernambuco

E-mail address: rodrigo.gondim.neves@gmail.com

Dipartimento di Matematica e Informatica, Università degli Studi di Catania, Viale A. Doria 5, 95125 Catania, Italy

E-mail address: frusso@dmi.unict.it 